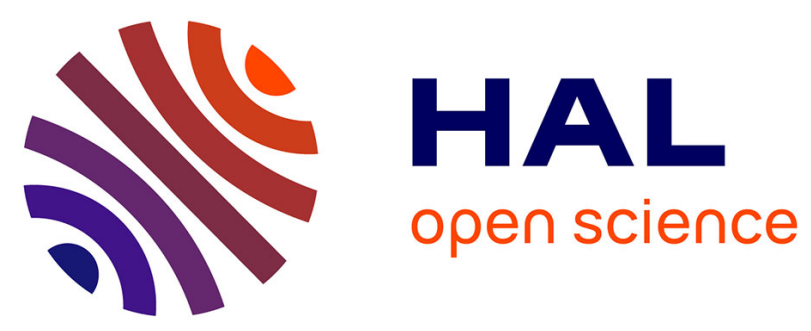

\title{
Two-stage electron acceleration by simultaneous stimulated Raman backward and forward scattering
}

Pierre Bertrand, A. Ghizzo, S. J Karttunen, T. J H Pättikangas, R. R E Salomaa, M. Shoucri

\section{- To cite this version:}

Pierre Bertrand, A. Ghizzo, S. J Karttunen, T. J H Pättikangas, R. R E Salomaa, et al.. Two-stage electron acceleration by simultaneous stimulated Raman backward and forward scattering. Physics of Plasmas, 1995, 2 (8), pp.3115-3129. 10.1063/1.871144 . hal-01804644

\section{HAL Id: hal-01804644 \\ https://hal.univ-lorraine.fr/hal-01804644}

Submitted on 1 Jun 2018

HAL is a multi-disciplinary open access archive for the deposit and dissemination of scientific research documents, whether they are published or not. The documents may come from teaching and research institutions in France or abroad, or from public or private research centers.
L'archive ouverte pluridisciplinaire HAL, est destinée au dépôt et à la diffusion de documents scientifiques de niveau recherche, publiés ou non, émanant des établissements d'enseignement et de recherche français ou étrangers, des laboratoires publics ou privés. 
Two-stage electron acceleration by simultaneous stimulated Raman backward and forward scattering

P. Bertrand, A. Ghizzo, S. J. Karttunen, T. J. H. Pättikangas, R. R. E. Salomaa, and M. Shoucri

Citation: Physics of Plasmas 2, 3115 (1995); doi: 10.1063/1.871144

View online: https://doi.org/10.1063/1.871144

View Table of Contents: http://aip.scitation.org/toc/php/2/8

Published by the American Institute of Physics

\section{Articles you may be interested in}

Theory of stimulated scattering processes in laser-irradiated plasmas

The Physics of Fluids 18, 1002 (1975); 10.1063/1.861248

Parametric instabilities of electromagnetic waves in plasmas

The Physics of Fluids 17, 778 (1974); 10.1063/1.1694789

Stimulated Raman forward scattering and the relativistic modulational instability of light waves in rarefied plasma

Physics of Fluids B: Plasma Physics 4, 2626 (1992); 10.1063/1.860178

The evolution of ultra-intense, short-pulse lasers in underdense plasmas

Physics of Plasmas 3, 2047 (1996); 10.1063/1.872001

Self-focusing and Raman scattering of laser pulses in tenuous plasmas

Physics of Fluids B: Plasma Physics 5, 1440 (1993); 10.1063/1.860884

Laser wakefield acceleration and relativistic optical guiding

Applied Physics Letters 53, 2146 (1988); 10.1063/1.100300

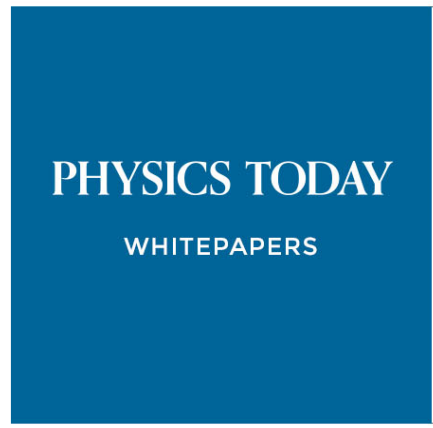

ADVANCES IN PRECISION MOTION CONTROL

Piezo Flexure Mechanisms and Air Bearings

\section{READ NOW}

PRESENTED BY PI 


\title{
Two-stage electron acceleration by simultaneous stimulated Raman backward and forward scattering
}

\author{
P. Bertrand and A. Ghizzo \\ Universite Henri Poincaré Nancy I, Laboratoire de Physique des Milieux lonisés-Centre National de la \\ Recherche Scientifique URA 835, B.P. 239, 54506 Vandoeuvre-lés-Nancy, France
}

S. J. Karttunen and T. J. H. Pättikangas
VTT Energy, P. O. Box 1604, FIN-02044 VTT, Finland

R. R. E. Salomaa

Department of Technical Physics, Helsinki University of Technology, FIN-02150 Espoo, Finland

M. Shoucri

Centre Canadien de Fusion Magnétique, Tokamak de Varennes, Varennes J3X 1S1, Québec, Canada

(Received 24 October 1994; accepted 15 March 1995)

\begin{abstract}
The coexistence of stimulated Raman forward and backward scattering of intense electromagnetic radiation, which can occur, for instance, in laser fusion plasmas, is investigated. The simultaneous Raman forward and backward scattering is shown to create an electrostatic field structure which is exceptionally efficient in producing highly relativistic electrons. The mechanism of the electron acceleration is analyzed both by Vlasov-Maxwell simulations with self-consistent fields and by test particle calculations with prescribed electrostatic fields. The Vlasov-Maxwell simulations reveal that the two plasma waves generated by the backward and forward scattering are spatially separated, and thus form a two-stage electron "accelerator." (c) 1995 American Institute of Physics.
\end{abstract}

\section{INTRODUCTION}

In recent years, the stimulated Raman scattering (SRS) has been studied extensively because SRS is able to produce fast electrons in laser-fusion. Even a small number of energetic electrons can cause severe preheating of the pellet core and prevent the efficient compression of the fuel capsule. In plasmas with short scale lengths, the observed SRS-levels are typically very low. However, with reactor-size pellets and longer laser pulses the interaction regions of the stimulated Raman scattering will grow considerably leading to an enhanced amount of Raman scattering ${ }^{1,2}$ and to an increasing number of fast electrons. In magnetic fusion, it was recently suggested that the so-called beat-wave process ${ }^{3-7}$ or the stimulated Raman scattering ${ }^{8}$ in the microwave region could be applied to current drive in tokamaks. The beat-wave process differs from the stimulated Raman scattering only by the initial and the boundary conditions. In current drive, the fast electrons are beneficial because they are almost collisionless and the current is easy to sustain.

The Raman scattered electromagnetic wave may propagate in backward or forward direction with respect to the pump wave. In both forward (SRS-F) and backward Raman scattering (SRS-B), the electron plasma waves travel forward but their phase velocities may differ considerably. Usually in laser-plasma experiments, SRS-B dominates over SRS-F because of its larger gain. In well underdense high-temperature plasmas, however, the Landau damping of the electron plasma wave strongly limits the growth of SRS-B. Under these conditions, stimulated Raman forward scattering can be comparable or even dominate over backscattering. In some cases, solely SRS-F is excited. ${ }^{9-11}$ The wavelength of the plasma wave is considerably longer in SRS-F than in SRS-B, and therefore the Landau damping is much weaker in SRS-F. This compensates the small gain of the Raman forward scattering so that an interesting competition between SRS-B and SRS-F is to be expected. The parameters corresponding to reactor-grade inertial confinement fusion experiments and to SRS current drive in tokamak fusion reactors lie in the intermediate region where both processes can occur simultaneously. ${ }^{12,13}$ For the fast electron current drive the coexistence of SRS-F and SRS-B provides a good method for maximizing the fast electron production.

The phase velocity of the plasma wave created in SRS-B is lower and closer to the electron thermal velocity than the phase velocity of the SRS-F plasma wave. Therefore, the intense SRS-B plasma wave can interact with a fairly large number of bulk electrons which get trapped by the wave potential. If the amplitude of the SRS-F plasma wave is so large that the trapping regions of the two plasma waves overlap in velocity space, numerous ultrafast electrons may be created. The role of SRS-B is to extract bulk electrons, and the role of SRS-F is to give them a further energy boost.

A simple model ${ }^{13}$ based on coupled mode theory predicts that in a homogeneous plasma slab, SRS-B and SRS-F are localized in spatially distinct regions: SRS-B in the front and SRS-F in the rear part of the slab. Therefore, the plasma waves excited by SRS-B and SRS-F are also localized in these regions, respectively. The electrons preaccelerated by SRS-B propagate deeper inside the plasma and are there further accelerated by SRS-F. Hence, the two wave packets do not have to overlap spatially-the overlapping of the trapping regions has to occur only in the velocity space. Some preliminary results on the two-stage acceleration were reported in a brief communication, Ref. 14. The results show clearly the qualitative difference of two-stage acceleration as compared to single field acceleration which takes place when only SRS-F is present. ${ }^{9.15}$

In this paper, we investigate in detail the above idea by 
Vlasov-Maxwell simulations and test particle calculations. The conditions for simultaneous Raman backward and forward scattering are considered in Sec. II by using a simplified envelope model. The Vlasov-Maxwell code and the simulation parameters are described in Sec. III. The Vlasov simulations in Sec. IV demonstrate the localization of SRS-B and SRS-F in different regions of the plasma and the generation of a large amount of ultrafast electrons. Test particle calculations with prescribed electrostatic fields in Sec. $V$ give further insight into the acceleration mechanism. The motion of test particles in the self-consistent electrostatic field generated by SRS is described in Sec. VI. Finally, the results are summarized and discussed in Sec. VII.

\section{SIMULTANEOUS BACKWARD AND FORWARD SRS}

\section{A. Conditions for simultaneous SRS-B and SRS-F}

In this paper, we assume a homogeneous plasma so that the wave numbers of the plasma waves do not change during the propagation. The model is expected to describe well stimulated Raman scattering in a weakly inhomogeneous plasma. Strong inhomogeneities change the situation to some extent because Raman scattering generates scattered waves with a wide range of frequencies. By considering homogeneous plasma, we shall be able to keep the model simple enough so that the different physical processes remain easily tractable.

Coupling between SRS-B and SRS-F is caused, e.g., by the common pump wave and indirectly by wave-particle interactions. In an inhomogeneous plasma, an additional coupling mechanism is possible: ${ }^{16,17}$ As the SRS-B plasma wave propagates from its generation region towards denser plasma its wave vector becomes smaller and it may become resonant with a local SRS-F process. In well underdense plasmas at high temperatures, the heavy Landau damping does, however, efficiently prevent the propagation of the SRS-B plasma wave and hence its coupling with SRS-F.

When both of the Raman processes occur simultaneously, there are three electromagnetic modes, i.e., the pump wave $E_{0}\left(k_{0}, \omega_{0}\right)$, the backscattered wave $E_{-}\left(k_{-}, \omega_{-}\right)$and the forward scattered wave $E_{+}\left(k_{+}, \omega_{+}\right)$. Two electrostatic plasma waves are excited: $E_{B}\left(k_{B}, \omega_{B}\right)$ and $E_{F}\left(k_{F}, \omega_{F}\right)$, where the subscripts $B$ and $F$ refer to backward and forward scattering, respectively. These waves obey approximately the linear dispersion relations

$$
\begin{aligned}
& \omega_{ \pm, 0}^{2}=\omega_{p}^{2}+k_{ \pm .0}^{2} c^{2}, \\
& \omega_{B, F}^{2}=\omega_{p}^{2}+3 k_{B, F}^{2} v_{e}^{2},
\end{aligned}
$$

where $\omega_{p}$ is the plasma frequency, $c$ is the velocity of light in vacuum and $v_{e}=\left(T_{e} / m_{e}\right)^{1 / 2}$ is the thermal velocity of electrons. Equations (1)-(2) together with the phase-matching conditions

$$
\begin{aligned}
& \omega_{0}=\omega_{+}+\omega_{F}=\omega_{-}+\omega_{B}, \\
& k_{0}=k_{+}+k_{F}=k_{-}+k_{B},
\end{aligned}
$$

determine the wave numbers and the frequencies of various modes once the pump wavelength, the plasma density and

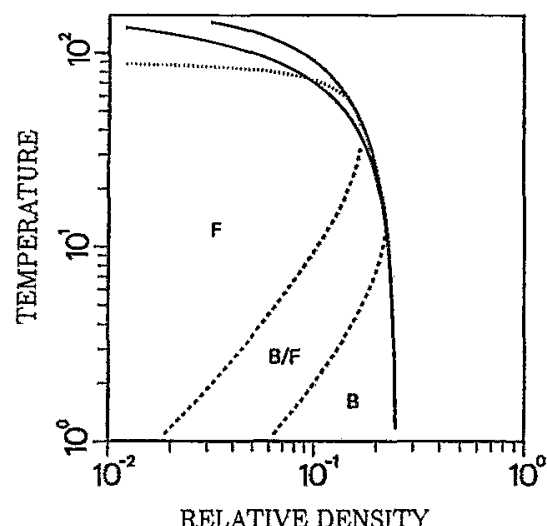

FIG. 1. Parameter regions for SRS-B and SRS-F in the $\left(n_{e}, T_{e}\right)$ plane. Under the solid lines SRS is possible. The dashed curves correspond to $k_{B} \lambda_{D}=0.3$ and $k_{B} \lambda_{D}=0.8$. SRS-B dominates in region " $\mathrm{B}$," SRS-F dominates in region " $F$ " and both processes coexist in region " $B / F$." Temperature is given in $\mathrm{keV}$ and relative density in $n_{\mathrm{e}} / n_{\mathrm{cr}}$

the electron temperature are given. A good approximation for the wave numbers of the plasma waves at a finite electron temperature is ${ }^{18}$

$$
\begin{aligned}
\frac{k_{B, F}}{k_{0}}= & \left\{1 \pm\left(\frac{1-2 \sqrt{N}}{1-N}\right)^{1 / 2}\left[1-\frac{3}{2}\left(\frac{v_{e}}{c}\right)^{2}\right.\right. \\
& \left.\left.\times \frac{(2-\sqrt{N})(1-\sqrt{N})}{1-2 \sqrt{N}}\right]\right\} / \\
& {\left[1+3\left(\frac{v_{e}}{c}\right)^{2}\left(\frac{1}{\sqrt{N}}-1\right)\right], }
\end{aligned}
$$

where $N=n_{e} / n_{\mathrm{cr}}$ and the " + " and " - " signs are for SRS-B and SRS-F, respectively.

The difference in the gain lengths of SRS-F and SRS-B is caused by the differences in the wave numbers of the excited plasma waves. The basic gain lengths are ${ }^{19,9}$

$$
L_{g B, F}=4 \sqrt{3}\left(\frac{c}{v_{0}}\right)\left|\frac{k_{-,+}}{k_{B, F}}\right|^{1 / 2} \lambda_{D}
$$

where $v_{0}=\left|q_{e} E_{0}\right| / m_{e} \omega_{0}$ is the electron quiver velocity in the pump field. The first subscripts should be chosen for SRS-B and the second ones for SRS-F, respectively.

Figure 1 shows the parameter regions for stimulated $\mathrm{Ra}$ man forward and backward scattering determined from the phase-matching relations. The two solid lines define the regions where Raman scattering can take place. In cold plasmas, one finds the usual condition $n_{e} / n_{\mathrm{cr}} \leqslant 0.25$. In the narrow strip between the two border lines, both solutions of the phase-matching relations correspond to forward scattering. The region where Raman scattering can occur has been divided into three domains depending on the Landau damping of the SRS-B plasma wave. At high densities $\left(n_{e} / n_{\mathrm{cr}}>0.1\right)$ and moderate temperatures $\left(T_{e}<5 \mathrm{keV}\right)$, the SRS-B plasma wave is weakly damped, and the backscattering dominates because of its higher gain. In Fig. 1, this region is labeled by " $B$ "; its left border line is determined by the condition $k_{B} \lambda_{D}<0.3$. At low densities and high temperatures, Landau damping suppresses Raman backscattering completely and the forward scattering dominates. This region has been la- 
beled by "F," and its border line is determined by the condition $k_{B} \lambda_{D}>0.8$. Only at extreme temperatures, the damping of the SRS-F plasma wave becomes excessive as is evidenced by the dotted line in the figure, above which $k_{F} \lambda_{D}>0.8$. In the intermediate region labeled " $\mathrm{B} / \mathrm{F}$," where $0.3<k_{B} \lambda_{D}<0.8$, both Raman processes can coexist.

The plasma conditions for simultaneous Raman backward and forward scattering correspond to those expected in underdense corona of reactor-size pellets of inertial confinement fusion. Electron temperatures clearly exceeding typical corona temperatures are found in laser produced filaments where the laser intensity is also enhanced. A significant fraction of Raman converted energy could be generated in these filaments. In the presence of filamentation, the transverse density gradient $\left(\nabla n_{e} \perp \mathbf{k}_{0}\right)$ may be steeper and more important than the longitudinal gradient $\left(\nabla n_{e} \| \mathbf{k}_{0}\right)$ in terms of scattered spectrum. Then it is also possible that all regions shown in Fig. 1 are present simultaneously.

The competition between SRS-B and SRS-F is also relevant in SRS current drive of tokamak plasmas by using millimeter waves. In tokamaks, the electron temperatures exceed $10 \mathrm{keV}$ and the electron density should be around $0.1 n_{\mathrm{cr}}$ in order to achieve a reasonable energy conversion to electrostatic modes.

\section{B. Simple envelope model}

In our previous simulations on pure $S R S-F,{ }^{9}$ calculations based on the approximation of slowly varying envelopes turned out to explain satisfactorily many features of the full solutions-in particular when nonlinear detunings and the anti-Stokes wave were included. The steady state analysis of simultaneous SRS-F and SRS-B performed in Ref. 13 also assumed slowly varying envelopes of the fields. The envelope equations can be written as

$$
\begin{aligned}
& \left(\frac{\partial}{\partial t}+v_{g 0} \frac{\partial}{\partial x}\right) a_{0}=-K_{B} a_{-} a_{B}-K_{F} a_{+} a_{F}, \\
& \left(\frac{\partial}{\partial t}-v_{g}-\frac{\partial}{\partial x}\right) a_{-}=K_{B} a_{0} a_{B}^{*}, \\
& \left(\frac{\partial}{\partial t}+v_{g} \frac{\partial}{\partial x}\right) a_{+}=K_{F} a_{0} a_{F}^{*}, \\
& \left(\frac{\partial}{\partial t}+v_{g B} \frac{\partial}{\partial x}+\Gamma_{B}\right) a_{B}=K_{B} a_{0} a_{-}^{*}, \\
& \left(\frac{\partial}{\partial t}+v_{g F} \frac{\partial}{\partial x}+\Gamma_{F}\right) a_{F}=K_{F} a_{0} a_{+}^{*} .
\end{aligned}
$$

The normalization of the fields is such that the squares of the amplitudes represent the action densities of the waves, i.e., $\left|a_{j}\right|^{2}=\varepsilon_{0}\left|E_{j}\right|^{2} / \omega_{j}(j=0,+,-)$ and $\left|a_{j}\right|^{2}=\left|E_{j}\right|^{2} / 2 \omega_{j}$ $(j=B, F)$. We shall further simplify the notation by defining $a_{j}$ as the relative amplitude $a_{j} / a_{0}(0,0)$ where $a_{0}(0,0)$ is the input pump wave action amplitude. We have assumed that the fields are plasma normal modes, and thus their detunings vanish. Furthermore, we have neglected the damping of electromagnetic waves and ignored nonlinear frequency shifts of the plasma waves. These simplifications are easily relaxed when necessary. The coupling coefficients are given by

$$
K_{B, F}=\frac{q_{e}}{2 m_{e}}\left(\frac{\omega_{B, F}}{2 \varepsilon_{0} \omega_{0} \omega_{-,+}}\right)^{1 / 2}\left|k_{B, F}\right| .
$$

In the presence of an external magnetic field which is parallel to the wave vectors of the waves the coupling coefficients can be found from Refs. 9, 20, and 21 .

We shall not enter here into a systematic study of the temporal behavior of Eqs. (7)-(11). Figure 2 gives examples of numerically solved steady state amplitude distributions for various backreflection coefficients $r_{-}=\left|a_{-}(0)\right|^{2}$ and input noise levels $\varepsilon_{+}=\left|a_{+}(0)\right|^{2}$. The dimensionless spatial coordinate is $x / L_{g B}$. The plasma parameters correspond to those of the Vlasov-Maxwell simulation in Sec. IV. Somewhat surprisingly, the main features of the curves are explainable by a simple model where the two processes, SRS-B and SRS-F, are fully decoupled. At second thought, this simplification is, of course, justifiable by the fact that the two processes are clearly spatially separated as evidenced by Fig. 2 . In other words, $\left|a_{+}\right|$is negligible in the front end of the plasma where $\left|a_{-}\right|$attains appreciable values and, vice versa, $\left|a_{-}\right|$is small near the rear end of the plasma where $\left|a_{+}\right|$ becomes comparable to $\left|a_{0}\right|$.

Let us first recall some basic results of SRS-B. In a homogeneous plasma, Raman backscattering is an absolute instability only when the (approximate) condition

$$
r_{-} \leqslant 1-\beta_{B}^{2} / 4
$$

holds. ${ }^{22}$ The normalized damping coefficient is given by $\beta_{B}=L_{g B} \Gamma_{B} / v_{g B}$ and $L_{g B}$ is the gain length of SRS-B, Eq. (6). The minimum length required for the onset of the instability is

$$
\frac{L}{L_{g B}}=\frac{\pi}{2}+\arcsin \frac{\beta_{B}}{2}
$$

which is valid for $r_{-} \rightarrow 0 .^{23}$ For finite values of $r_{-}$we can use for the characteristic interaction length-i.e., for the length where the backscattered field goes to zero-the approximate expression $^{22}$

$$
\frac{L}{L_{g B}}=\left[K\left(r_{-}^{1 / 2}\right)+\frac{1}{2} F\left(\varphi \mid r_{-}^{1 / 2}\right)\right]\left[1-\frac{\beta_{B}^{2}}{4\left(1-r_{-}\right)}\right]^{-1 / 2},
$$

where $F(\varphi \mid m)$ is the elliptic integral of the first kind and $K$ the corresponding complete integral. The argument $\varphi$ is defined as

$$
\varphi=\arctan \frac{2 \beta_{B}\left(1-r_{-}-\beta_{B}^{2} / 4\right)^{1 / 2}}{\left(1-r_{-}\right)^{1 / 2}\left(1-r_{-}-\beta_{B}^{2} / 2\right)} .
$$

Equation (13) thus gives the maximum backreflection obtainable in steady state, when noise is ignored in SRS-B. Equation (15), or Eq. (14) in the limit $r_{-} \ll 1$, gives an estimate for the position of the first zero of $a_{-}$in Fig. 2.

The steady state equations for SRS-B satisfy the conservation law $\left|a_{0}\right|^{2}-\left|a_{-}\right|^{2}=1-r_{-}=\left|a_{0}(L)\right|^{2}$ when the damping of the electromagnetic waves is negligible. If SRS-B and SRS-F are assumed to appear in two successive 

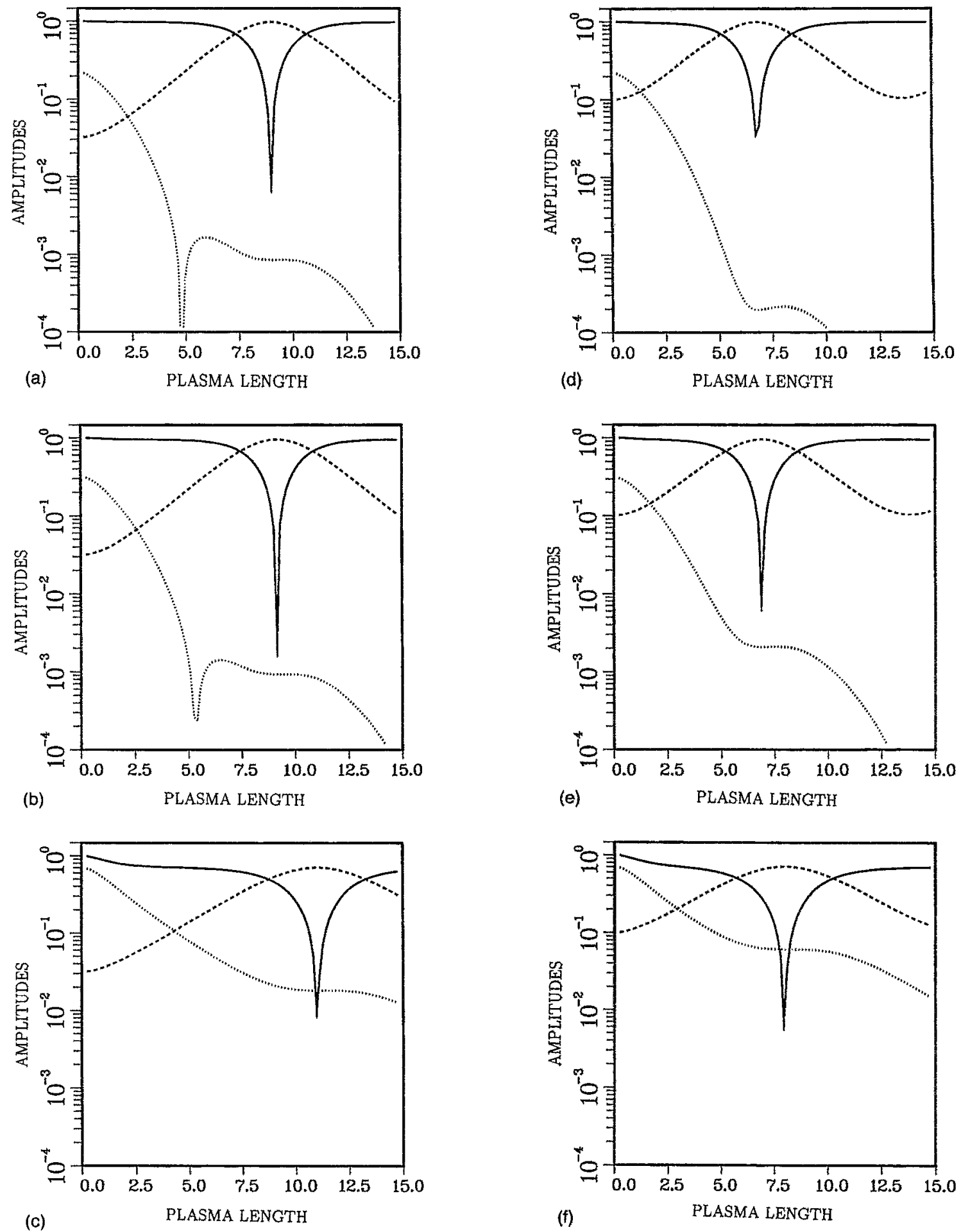

FIG. 2. The normalized steady state electromagnetic field amplitudes $\left|a_{0}\right|$ (solid line), $\left|a_{+}\right|$(dashed line), and $\left|a_{-}\right|$(dotted line) as a function of plasma length (in gain lengths $L_{g B}$ of SRS-B). In the frames on the left, the input noise level is $\left|a_{+}(0)\right|^{2}=\varepsilon_{+}=10^{-3}$ and the back reflectivity varies as $\left|a_{-}(0)\right|^{2}=r_{-}=0.05,0.10$, and 0.50 from (a) to (c). In the frames on the right, (d) to (f), the noise level is increased to $\varepsilon_{+}=10^{-2}$. The normalized damping rates are $\beta_{B}=1.635$ and $\beta_{F} \approx 0$, and the gain length ratio equals $\alpha=0.548$.

spatial regions, then SRS-F does not experience the full input pump intensity $\left|a_{0}(0)\right|^{2}=1$ but the reduced value $\left|a_{0}(L)\right|^{2}=1-r_{-}$.

The exact solution of SRS-F alone is expressable in terms of elliptic integrals in the case of negligible damping.
The conversion length, i.e., the length at which $a_{0}(L)=0$, is given by

$$
\frac{L}{L_{g B}}=\frac{K\left[\left(1+\varepsilon_{+} /\left|a_{0}(0)\right|^{2}\right)^{-1 / 2}\right]}{\alpha\left(1+\left.\varepsilon_{+}|| a_{0}(0)\right|^{2}\right)^{1 / 2}}
$$


where $\alpha=L_{g B} / L_{g F}$. Referring to the discussion above we have kept the option of having $\left|a_{0}(0)\right|^{2}<1$ because of the presence of SRS-B in front of the generation region of SRS-F. In the limit $\left|a_{0}(0)\right|^{2}=1-r_{-} \gg \varepsilon_{+}$, we find ${ }^{24}$

$$
\frac{L}{L_{g B}}=\frac{1}{2 \alpha\left(1-r_{-}+\varepsilon_{+}\right)^{1 / 2}} \ln \left[\frac{16}{\varepsilon_{+}}\left(1-r_{-}+\varepsilon_{+}\right)\right] .
$$

The conversion length becomes infinite in the limit $\varepsilon_{+} \rightarrow 0$, which implies that SRS-F is a convective instability. The conversion length, Eq. (17) or (18), gives the position of the deep dip occurring in the pump intensity curves in Fig. 2.

In Figs. 2(a) to 2(c) the observed positions of the pump minima, 9.01 (9.03), $9.12(9.20), 10.95$ (11.58), for $\varepsilon_{+}=10^{-3}$ and $r_{-}=0.05,0.10$, and 0.50 , respectively, agree very well with the values calculated from Eq. (18) which are given in parentheses. This is also true for the higher input noise level $\varepsilon_{+}=10^{-2}$ : the minima appear at $6.77(6.83), 6.90$ (6.97), and 7.96 (8.57) in Figs. 2(d) $-2(\mathrm{f})$.

The characteristic SRS-B interaction length at which $a_{-} \rightarrow 0$ is 4.78 (5.68) and 5.38 (6.10) in Figs. 2(a) and 2(b), respectively, which agree reasonably well with the values given in parentheses which were calculated by using Eq. (15). The characteristic length increases with $r_{-}$, and when it approaches the conversion length of SRS-F (the dip in $\left|a_{0}\right|$ curves) the zero in the backscatter amplitude curve $\left|a_{-}\right|$becomes a turning point [see Figs. 2(a)-2(c)]. This indicates that SRS-B, disturbed by SRS-F, turns from an absolute instability into a convective one. This occurs at $r_{-}=0.047,0.205$, and 0.276 , when $\varepsilon_{+}=10^{-2}, 10^{-3}$, and $10^{-4}$, respectively. For larger values of $r_{-},\left|a_{-}\right|^{2}$ may still truly vanish or become practically negligible, but the required plasma lengths increase rather rapidly. For instance the zero of $\left|a_{-}\right|$escapes the displayed region of Fig. 2 when $r_{-} \approx 0.25$ and $\varepsilon_{+}=10^{-3}$ [cf. also Fig. 2(c)]. According to (13), a pure SRS-B would be an absolute instability only when $r_{-}<0.332$ for the assumed value of $\beta_{B}$.

It is worth pointing out that the steady state curves in Fig. 2 do not necessarily represent true stationary solutions. The reason is, of course, that the prechosen reflectivity $r_{-}$, the length of the plasma slab and the noise levels do not usually form a fully consistent set. The appearance of several zeros of $\left|a_{-}\right|$suggests that the solution contains higher order, unstable eigenmodes. Here, we do not perform any stability analysis of the steady state solutions.

SRS-F is rather modestly affected by SRS-B. In contrast to this, SRS-F can influence remarkably the absolute SRS-B instability. In a pure three-wave interaction, the maximum reflectivity of an absolute SRS-B instability is limited by the condition (13). The pump intensity is reduced by SRS-F and consequently the maximum value of $r_{-}$drops from the value 0.332 . When the conversion length (17) approaches the threshold length (14), SRS-B is expected to disappear. For the parameters of our Vlasov-Maxwell simulation this would require a rather high input noise intensity $\varepsilon_{+} \approx 0.130$.

Although we shall not enter a detailed discussion of the temporal solutions of (7)-(11), it is still instructiveespecially as regards the interpretation of the simulation results - to consider some characteristics of those equations.

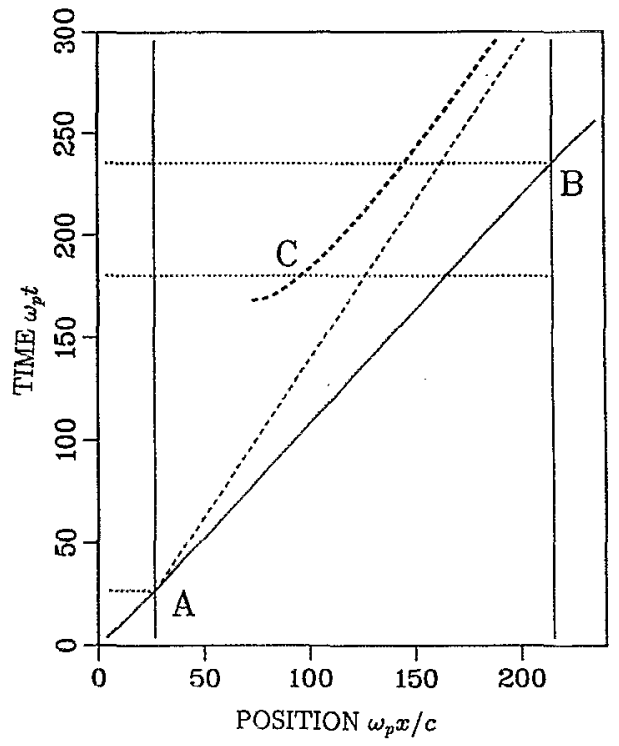

FIG. 3. The main characteristics of the Vlasov-Maxwell simulation. The plasma slab lies between the vertical lines. The solid and the dashed lines describe the fronts of the pump wave and the forward scattered wave, respectively. The dashed curve displays the position where $\left|a_{+}\right|$has grown approximately tenfold from its initial noise level.

An example set of these is displayed in Fig. 3. Notice that the figure is drawn using the simulation units. In the VlasovMaxwell simulation, the group velocities of the waves (in units of $c$ ) are $0.902,-0.474,0.652,0.132$, and 0.0715 for the pump, backward and forward scattered electromagnetic waves, SRS-B and SRS-F plasma waves, respectively.

In Fig. 3, the plasma slab surrounded on both sides by voids lies between the two vertical lines. The pump wave front represented by the solid line enters the plasma at point $A$ and leaves it at point $B$. The dashed line gives the front of the forward scattered wave propagating at a speed 0.652 . The pump wave has propagated to a depth $5 L_{g B}$ by the time 50.7 . The backward scattered wave will do the same distance within a time 45.9 and as the growth time of the instability is short enough (between 5 and 25 depending on the approximation), SRS-B would be almost fully developed by $\omega_{p} t \approx 100$. Thus as a first approximation for $\omega_{p} t>100$ SRS-B is stationary and it occurs in the region $\omega_{p} x / c<50$.

In the following, we neglect the damping of the forward plasma wave $\left(\Gamma_{F}=0\right)$ and its propagation $\left(v_{g F}=0\right)$, and ignore pump depletion, i.e., we put $a_{0}(x, t)=a_{0} \theta\left(v_{g 0} t-x\right)$ where $\theta(x)$ is the Heaviside step function. Then the linearized solution for $a_{+}$reads

TABLE I. Parameters of the waves in simultaneous SRS-B and SRS-F.

\begin{tabular}{lcccc}
\hline & $\omega_{j} / \omega_{p}$ & $k_{j} c / \omega_{p}$ & $v_{\mathrm{ph}} / c$ & $p_{\mathrm{ph}} / m_{e} c$ \\
\hline Pump wave $E_{0}$ & 2.36 & 2.13 & & \\
Stokes wave $E_{-}$in SRS-B & 1.14 & 0.54 & & \\
Plasma wave $E_{B}$ in SRS-B & 1.19 & 2.68 & 0.45 & 0.50 \\
Stokes wave $E_{+}$in SRS-F & 1.32 & 0.86 & & \\
Plasma wave $E_{F}$ in SRS-F & 1.05 & 1.28 & 0.82 & 1.42 \\
Anti-Stokes wave $E_{A S}$ in SRS-F & 3.41 & 3.41 & & \\
\hline \hline
\end{tabular}


$\omega_{p} t=180$
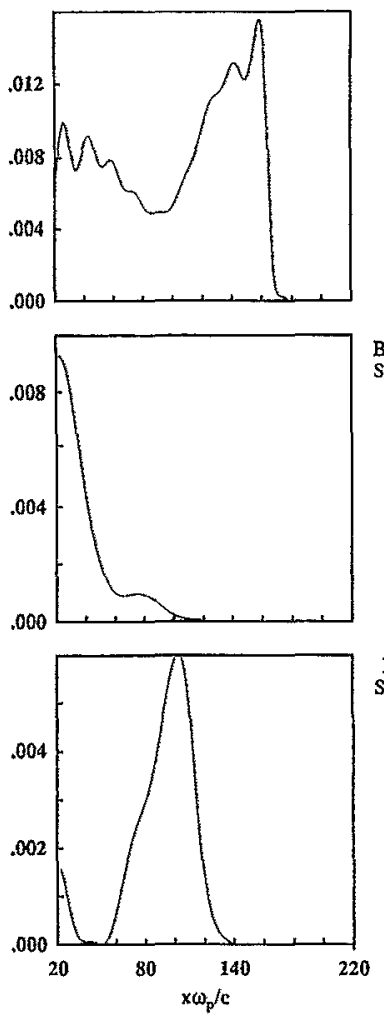

FIG. 4. Spatial dependence of the envelopes of the pump wave and the backward and forward scattered waves in action density units at $\omega_{p} t=180$ and 260 .

$$
a_{+}(x, t)=a_{+}(0) \theta\left(v_{g+} t-x\right) \mathrm{I}_{0}\left(2 \kappa \sqrt{x\left(v_{g+} t-x\right)}\right),
$$

where $I_{0}$ is the modified Bessel function, $k=\left|K_{F} a_{0} / v_{g+}\right|$, and the boundary conditions $a_{+}\left(v_{g 0} t-x, t\right)=0$, $a_{+}(0, t)=a_{+}(0), \quad a_{F}\left(v_{g 0} t-x, t\right)=0$, and $a_{F}(0, t)=0$ are used. For these boundary conditions, the forward scattered field front is at $x=v_{g+} t$. The amplitude of the plasma wave, in the considered case, is given by

$$
\begin{aligned}
a_{F}(x, t)= & a_{+}(0)^{*} \theta\left(v_{g+} t-x\right)\left(\frac{v_{g+} t}{x}-1\right)^{1 / 2} \\
& \times I_{1}\left(2 \kappa \sqrt{x\left(v_{g+} t-x\right)}\right) .
\end{aligned}
$$

In the dashed curve of Fig. 3, we have $a_{+}(x, t) \approx 10 a_{+}(0)$, and thus it roughly gives the position of the maximum of the forward scattered SRS-F wave. For instance, at $\omega_{p} t=180$ (the middle horizontal dotted line) the field maximum would occur at the point $\mathrm{C}$ where $x \omega_{p} / c \approx 95$, which agrees well with the simulation result, see Fig. 4. Similarly all the present results based on the envelope model and its simplified solutions are applicable when analysing the gross features of the Vlasov-Maxwell simulations.

\section{VLASOV-MAXWELL SIMULATIONS}

Most of the simulations of Raman scattering in a plasma have been performed by using particle codes. ${ }^{12,25-27}$ The well-known particle-in-cell (PIC) techniques make it difficult to obtain an accurate enough description of the electron ac- celeration process. This is because in the PIC codes the number of simulation particles is too small to display the details of the phase space region where particle velocities are close to the phase velocities of the waves and where trapping occurs.

If more than one spatial dimension is involved, only the PIC codes have been practical so far because they are stable even when a fairly small number of simulation particles are used. When one spatial dimension will do, the Eulerian Vlasov codes have, however, been found to be a powerful tool for studying in detail the particle acceleration dynamics even at relativistic energies. In Eulerian Vlasov codes, one calculates the Vlasov fluid density in phase space without using simulation macro-particles. Such simulations have been performed both in conveniently short but idealized periodic plasmas $^{28}$ and in more realistic long open systems with external sources, ${ }^{29}$ which we characterize as "causal" systems. Particle acceleration has been investigated both in unmagnetized $^{29}$ and magnetized ${ }^{9}$ plasmas. Our need for an accurate description of the dynamics of accelerated electrons strongly suggests using Vlasov codes.

Let us consider a relativistic plane wave propagating in the $x$ direction with the electric field of the wave $\mathbf{E}$ in the $y$ direction and the magnetic field of the wave $\mathbf{B}$ in the $z$ direction. In a plasma, all quantities are only supposed to be functions of time and the spatial variable $x$. The oscillatory electric field $E_{y}$ entering the plasma produces quiver motion of the electrons which couples nonlinearly with the magnetic field $B_{z}$ producing a force parallel to the laser wave vector direction $x$. This so-called ponderomotive force drives a large-amplitude plasma wave in Raman scattering and beatwave process.

The plasma can be accurately described by the Vlasov equation for the electron distribution function $f\left(x, p_{x}, t\right)$ :

$$
\frac{\partial f}{\partial t}+\frac{p_{x}}{m_{e} \gamma} \frac{\partial f}{\partial x}+q_{e}\left(E_{x}+u_{y} B_{z}\right) \frac{\partial f}{\partial p_{x}}=0
$$

where $y=\left[1+\left(p_{x} / m_{e} c\right)^{2}\right]^{1 / 2}$ is the Lorentz factor and $p_{x}=m_{e} \gamma v_{x}$. In the perpendicular $y$ direction, we assume that monokinetic fuid description is valid. The two terms $u_{x} \partial u_{y} / \partial x$ and $u_{x} B_{z}$ exactly cancel each other, so that the relevant macroscopic equation for perpendicular fluid momentum $P$ reduces to:

$$
\frac{\partial \mathrm{P}_{y}}{\partial t}=q_{e} E_{y} .
$$

The ions are assumed to be immobile. The self-consistent longitudinal electric field $E_{x}$ is given by the Poisson equation, while the transverse electromagnetic fields $E_{y}$ and $B_{z}$ obey the Maxwell equations as discussed in Ref. 29. It must be stressed that the one-dimensional model is not an approximation but an exact subset of the full kinetic one-and-onehalf-dimensional ( $1 \frac{1}{2}$-D) Vlasov model. Using periodic boundary conditions, a very good agreement has been found between this simplified description and the full kinetic code in unmagnetized and magnetized plasmas.

We consider a homogeneous plasma slab of length $L$ surrounded by a void where an electromagnetic pump wave is launched at $x=0$. The length of the homogeneous plasma 
is $L=64 \lambda_{0}=188 \mathrm{c} / \omega_{p}$, where $\lambda_{0}$ is the pump wavelength in plasma. A void of length $L_{\mathrm{void}}=9 \lambda_{0}$ is on both sides of the plasma. The frequency of the pump wave is $\omega_{0}=2.36 \omega_{p}$ (i.e., $n / n_{\mathrm{cr}}=0.18$ ), and the amplitude of the quiver velocity is $v_{0} / c=0.1$. Initially, the plasma is homogeneous and Maxwellian with a thermal velocity $v_{\text {th }}=0.14 c$, corresponding to a temperature of $T_{e}=10 \mathrm{keV}$. These parameters are in the simultaneous SRS-B and SRS-F region of Fig. 1.

Since the Eulerian Vlasov code is essentially noiseless, the electron distribution was modified to include a smallamplitude forward-propagating electrostatic wave in order to trigger the SRS-B instability:

$$
\begin{aligned}
f\left(x, p_{x}, t=0\right)= & F\left(p_{x}-\varepsilon m_{e} v_{\text {th }} \cos k_{B} x\right) \\
& \times\left[1+\frac{\varepsilon v_{\mathrm{th}} k_{B}}{\omega_{B}} \cos k_{B} x\right]
\end{aligned}
$$

where

$$
F\left(p_{x}\right)=\frac{1}{\sqrt{2 \pi v_{\text {th }}}} \exp \left(-\frac{p_{x}^{2}}{2 m_{e}^{2} v_{\mathrm{th}}^{2}}\right) .
$$

The plasma parameters given above are in the optimum operating region for simultaneous SRS-B and SRS-F as can be verified from Fig. 1. The electron density and the pump intensity are chosen high in order to maximize the SRS growth rate and to reduce the computation time. The steep density gradient at the entrance into the plasma is sufficient for creating a perturbation that starts the Raman forward instability. This perturbation determines the noise level $\epsilon_{+}$ which was used in the envelope calculations of Sec. II B.

The frequencies and the wave numbers of the scattered electromagnetic and electrostatic waves can be solved from the dispersion relations (1) and (2) and the phase-matching conditions (3) and (4). The wave numbers and the frequencies of the various waves are given in Table I. Note that the linear Landau damping of the plasma wave excited by SRS-B is strong, $\Gamma_{B} / \omega_{p}=0.049$, in contrast to the wave excited by SRS-F which is almost undamped $\left(\Gamma_{F} / \omega_{p}=3.5 \times 10^{-6}\right)$. The phase velocities (momenta) of the plasma waves are $v_{\mathrm{phB}}=0.45 c\left(p_{\mathrm{phB}}=0.50 m_{e} c\right)$ and $v_{\mathrm{phF}}=0.82 c\left(p_{\mathrm{phF}}=1.42 m_{e} c\right)$. When the quiver velocity is $v_{0} / c=0.1$, the gain lengths of the Raman backward and forward instabilities are $L_{g B}=4.35 c / \omega_{p}$ and $L_{g F}=7.95 c / \omega_{p}$, respectively.

\section{RESULTS OF VLASOV-MAXWELL SIMULATIONS}

In the Vlasov-Maxwell simulation, the pump amplitude and the initial density perturbation of the plasma were chosen sufficiently large in order to ensure that pump depletion and simultaneous SRS-B and SRS-F occur within a reasonable time $\left(\omega_{p} t \simeq 180\right)$. The pump amplitude was $v_{0} / c=0.1$ and the initial perturbation was $\varepsilon=0.06$ which corresponds to a density perturbation of $\delta n_{e} / n_{e}=1.9 \times 10^{-2}$, see Eq. (23).

The action density envelopes of the electromagnetic fields are defined in terms of the energy density of the wave W:

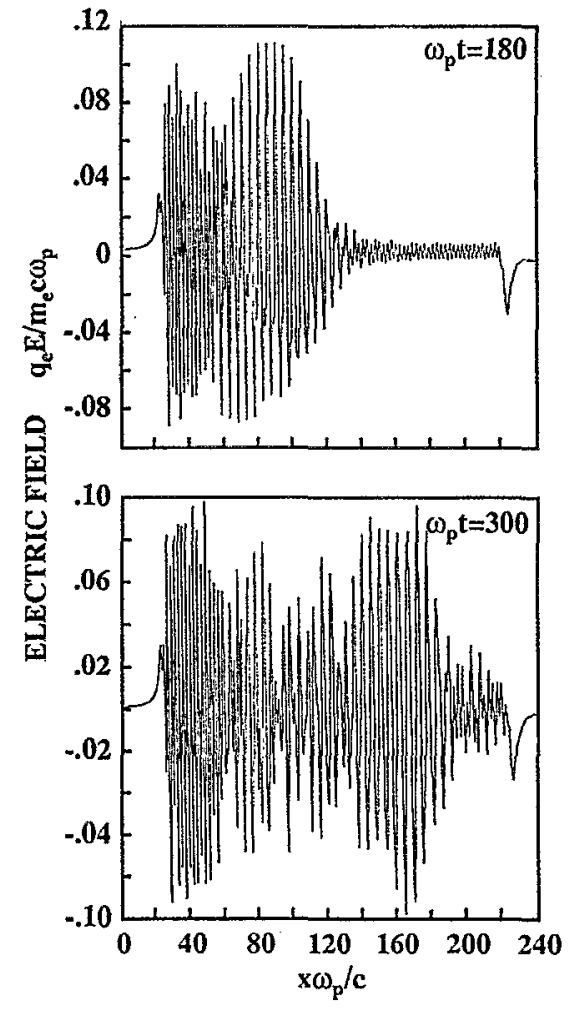

FIG. 5. Spatial dependence of the normalized electrostatic field $\left|q_{e}\right| E / m_{e} c \omega_{p}$ at $\omega_{p} t=180$ and 300 .

$$
A=\frac{W}{\omega}=\frac{1}{4 \omega}\left[\mu_{0}|H|^{2}+\epsilon_{0} E \frac{\partial \omega \epsilon_{r}}{\partial \omega} E^{*}\right]
$$

Here $\epsilon_{r}$ is the transverse $(r=0, \pm)$ or longitudinal $(r=F, B)$ relative dimensionless dielectric coefficient, $\epsilon_{0}$ is the vacuum permittivity, and $\mu_{0}$ is the vacuum permeability. The amplitudes of the appropriate electric and magnetic field components are denoted by $E$ and $H$, respectively. The envelopes were obtained from the simulation results by the Hilbert transform.

Figure 4 shows the envelopes of the action densities of the electromagnetic waves at $\omega_{p} t=180$ and 260. At $\omega_{p} t=180$, backward and forward scattering have grown to an appreciable level but the pump depletion is still incomplete. Later at $\omega_{p} t=260$, the action of the forward scattered field well exceeds that of the backscattered field, and the pump is completely depleted just behind the maximum of the forward action. In addition, modulations start to develop in the envelopes later in time. The action densities of the backward and forward scattered waves are localized and well separated. SRS-B takes place near the front edge of the plasma and SRS-F occurs deeper inside the plasma slab. The gross features in Fig. 4 can be understood by the simple envelope model of Sec. II B. For instance, the propagation front of the forward scattered wave coincides well with the predictions of Fig. 3. Similarly for SRS-B, the backscattered wave amplitude becomes negligible beyond $x>50$; the interaction length thus corresponds to about $5 L_{g F}$ and is in excellent agreement with Fig. 2. 



FIG. 6. Wave number spectra of the electromagnetic and the electrostatic field at $\omega_{p} t=180$.

The total electrostatic field at $\omega_{p} t=180$ and 300 is shown in Fig. 5. The two main Fourier components have very clear-cut features because they are localized and their wavelengths differ considerably. The backward field with the shorter wavelength appears in the front part of the plasma slab, and the forward field is located deeper inside the plasma. This is even more pronounced at the earlier time, before the onset of amplitude modulations. Later, saturation effects, wave-wave and wave-particle interactions start to play a role. At $\omega_{p} t=180$, the initial perturbation at the shorter wavelength is seen in the front of the leading edge of the pump wave $\left(x \omega_{p} / c>170\right)$.

The wave number spectra of the electromagnetic and electrostatic fields at $\omega_{p} t=180$ are illustrated in Fig. 6 . The narrow peaks in both spectra occur exactly at the positions calculated from the phase-matching conditions (see Table I). At $\omega_{p} t=300$, nonlinear effects become important, and the electrostatic spectrum broadens considerably. At the same time, the action density envelopes exhibit strong modulations (see Ref. 14).

In Fig. 7, the envelopes of the electrostatic field components are shown at two different times. The envelopes have been obtained by Hilbert transforming the fields shown in Fig. 5. The spatial separation of the electrostatic waves is very clear. At $\omega_{p} t=180$, the backward field extends from $x \omega_{p} / c=20$ to about 50 , and the forward wave is peaked around $x \omega_{p} / c=90$.

In Fig. 8, the fast electron generation is illustrated by a phase-space plot at two different times: $\omega_{p} t=250$ and 300 . Figure 8 also shows the separatrix momentum of marginally
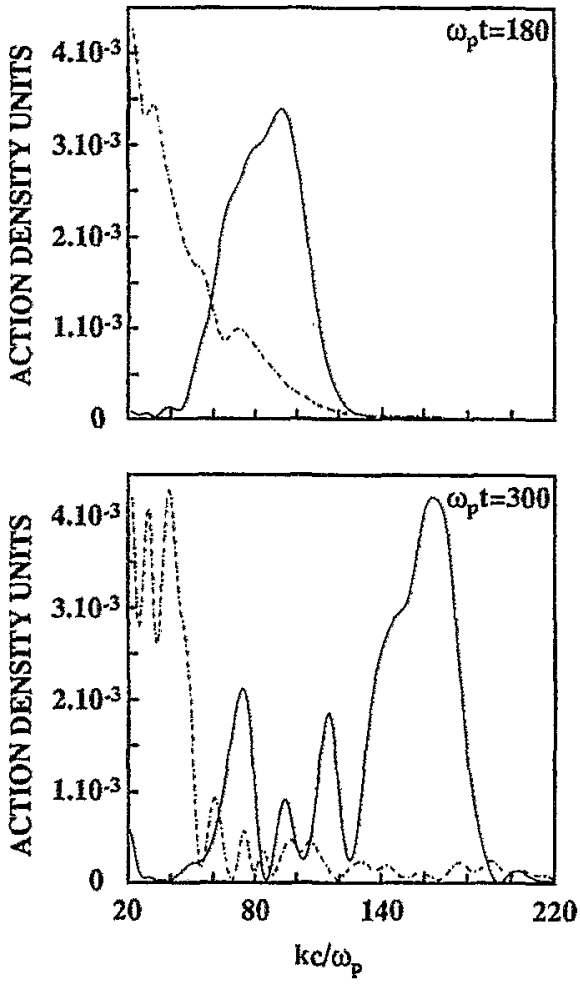

FIG. 7. Envelopes of the electrostatic fields at $\omega_{p} t=180$ and 300 .

trapped electrons for both electron plasma waves. It demonstrates that the overlapping of the trapping regions has to occur only in the velocity space and not necessarily locally. It is enough that the trapping region at the maximum of the SRS-B plasma wave overlaps with the trapping region of the maximum at the SRS-F plasma wave. Then the electrons accelerated by SRS-B in the front part of the plasma are further accelerated by SRS-F when they arrive in the central region of the plasma slab. At the rear end of the plasma, the electrostatic field becomes weaker, and therefore the decoupled energetic electrons are streaming freely as can be seen in the phase-space plot.

Figure 9 shows two distribution functions at $\omega_{p} t=300$ corresponding to spatial averages over the regions $26.5 \leqslant x \omega_{p} / c \leqslant 50$ and $120.5 \leqslant x \omega_{p} / c \leqslant 144$, respectively. In the front part, $26.5 \leqslant x \omega_{p} / c \leqslant 50$, the dominant waveparticle interaction is due to the plasma wave created by SRS-B. A plateau is formed in the distribution function of Fig. $9(\mathrm{a})$ around $\gamma_{\mathrm{phB}}-1=0.11$, where $\gamma_{\mathrm{phB}}$ is the Lorentz factor at $v_{\mathrm{phB}}$. In the center of the plasma, represented by the distribution function in Fig. 9(b), the plateau appears around $\gamma_{\mathrm{phF}}-1=0.75$. As the separatrices in the phase-space plot show, the trapping region extends from $\gamma-1=0.1$ to about 2.3 , which agrees well with the observed plateau region.

A key feature in the distribution functions in Fig. 9 is that the height of the plateau in the SRS-F dominated region is only one order of magnitude smaller than in the SRS-B region. This demonstrates that fast electron generation can be drastically increased when both the backward and the forward electrostatic fields are simultaneously present even though they are not spatially overlapping. 

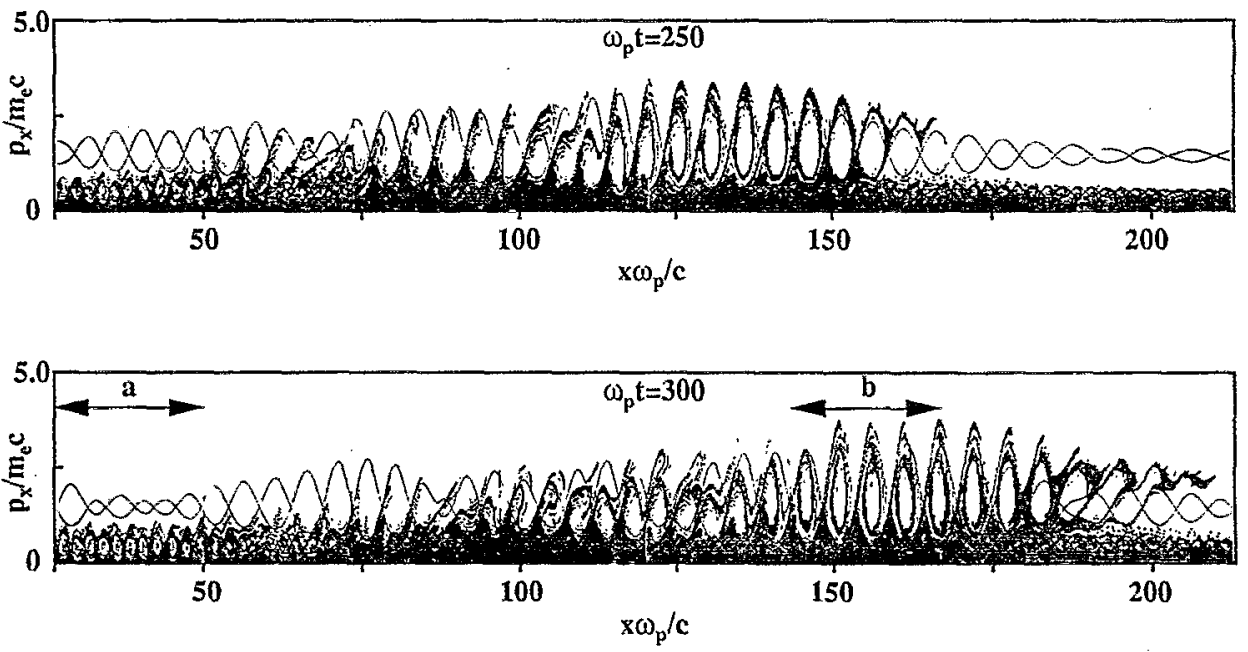

FIG. 8. Phase-space plot $\left(x, p_{x}\right)$ of the electron distribution function at $\omega_{p} t=250$ and 300 . The solid lines show the separatrix momentum of a marginally trapped electron. The markers "a" and " $b$ " refer to the spatially averaged electron distribution functions shown in Fig. 9.

The formation of the large hot tail in the distribution is illustrated in Fig. 10 which shows a window moving at the linear phase velocity $v_{\mathrm{phF}}$ of the fast SRS-F plasma wave computed from Eq. (2). Because of nonlinearities the actual phase velocity deviates somewhat from $v_{\mathrm{phF}}$ and thus the potential well marked by an arrow is not exactly stationary in the moving frame. At $\omega_{p} t=200$, the window is in the front part of the plasma slab where SRS-B is the strongest process. In this region, the slower SRS-B plasma wave feeds particles into the tail of the distribution, but the interaction of the
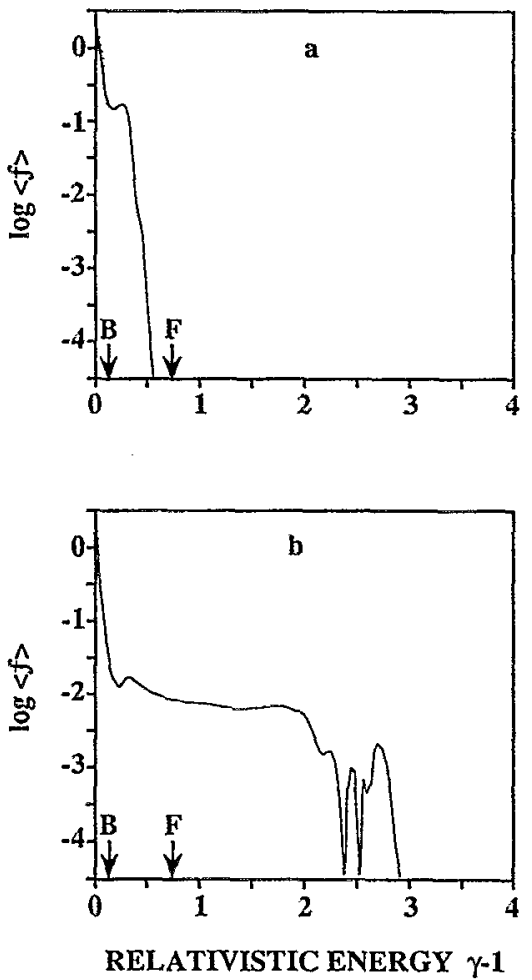

FIG. 9. Spatially averaged electron velocity distributions as a function of $\gamma-1$ at $\omega_{p} t=300$ corresponding to the regions " $\mathrm{a}$ " and " $\mathrm{b}$ " indicated in Fig. 8. The arrows (B,F) stand for the phase velocities of the plasma waves. particles and the SRS-F plasma wave is still almost negligible. Between $\omega_{p} t=220$ and 280 , the window and the potential wells of the faster plasma wave move to the region where SRS-F is strongest. During the propagation, the amplitude of the SRS-F plasma wave grows and it starts to trap electrons preaccelerated by the slower plasma wave. After $\omega_{p} t=320$, the moving window and the fast electrons arrive into the region where the amplitude of the SRS-F plasma wave is gradually decreasing. Some of the fast electrons become untrapped and stream freely with a velocity larger than the phase velocity of the faster wave.

We have shown that our Eulerian Vlasov code is able to give a precise description of the phase-space dynamics with striking agreement with the simple orbit theory of separatrices. The wave-particle interaction in simultaneous SRS-B and SRS-F is strong in spite of the large phase velocity of the SRS-F plasma wave: $v_{\mathrm{phF}}=0.82 c$. The reason for this is Raman backscattering which enhances the wave-particle interaction because of its smaller phase velocity. When the lower trapping velocity computed from the potential induced by SRS-F approaches $v_{\mathrm{phB}}$, strong wave-particle interaction takes place because SRS-B has increased the electron density around $v_{\mathrm{phB}}$. Simultaneously, the pump wave is depleted, which leads to an efficient energy transfer to the interacting electrons.

An analogous process has already been observed earlier in a magnetized plasma ${ }^{9}$ in conditions relevant to the Raman or beat-wave current drive in reactor grade tokamak plasmas. In those simulations, a second slower plasma wave was excited due to multiple scattering of the pump wave, which occurred during generation of the second Stokes wave. The simultaneous appearance of two plasma waves with different phase velocities was found to increase the wave-particle interaction.

\section{TEST ELECTRONS IN PRESCRIBED FIELDS}

To understand the behavior of individual electrons, we have studied the staged acceleration of electrons by test par- 

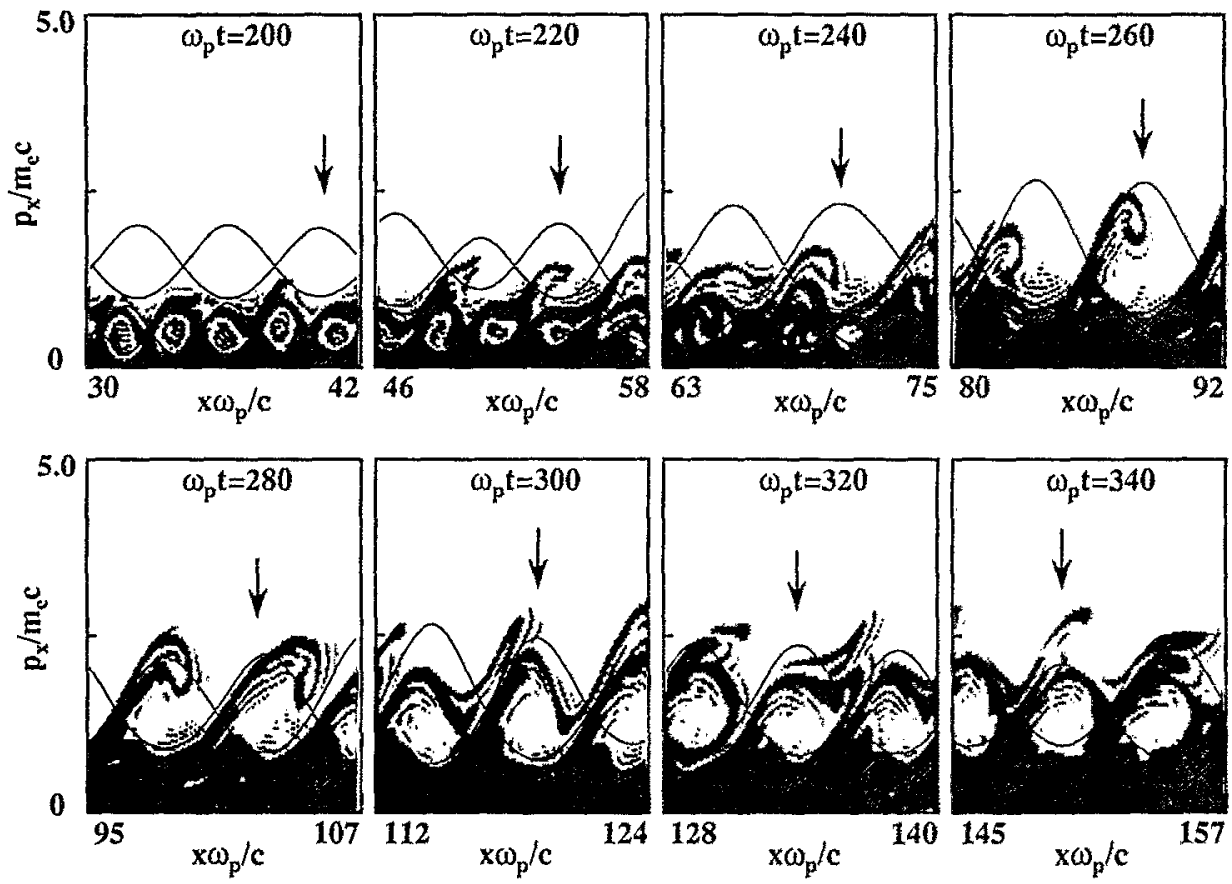

FIG. 10. Phase-space window moving at the phase velocity of the SRS-F plasma wave when both SRS-B and SRS-F occur. The solid lines show the separatrix momentum of a marginally trapped electron. One of the potential wells has been marked by an arrow.

ticle simulations in prescribed spatially separated fields. We have chosen the fields so that they resemble the plasma waves generated by SRS-B and SRS-F. We have solved numerically the equations of motion of an electron

$$
\begin{aligned}
& \frac{d x}{d t}=v, \\
& \frac{d p}{d t}=q_{e} E(x, t) .
\end{aligned}
$$

The two-component electrostatic field $E$ is defined as

$$
E(x, t)=\sum_{i=F, B} E_{i}(x) \cos \left(k_{i} x-\omega_{i} t+\varphi_{i}\right),
$$

where Gaussian envelopes

$$
E_{i}(x)=E_{i} \exp \left[-\left(x-x_{i}\right)^{2} / w_{i}^{2}\right]
$$

have been assumed. The test electrons have the initial momenta $p_{\text {in }}$ at $x=0$, and the final momenta $p_{\text {out }}$ at $x=L$ were calculated. The frequencies and the wave numbers in (28) were chosen to correspond to the SRS-B and SRS-F plasma waves of the Vlasov simulation. The test particle calculations are analogous to the non-relativistic calculations performed by Fuchs $e t$ al. ${ }^{30}$ for a single wave packet.

Inspired by the Vlasov simulations, we chose the two Gaussian fields centered at $x_{B}=L / 4$ and $x_{F}=3 L / 4$, where $L \omega_{p} / c=188$ was the length of the simulation box. The widths of the Gaussians were $w_{B, F} \omega_{p} / c=15$, and the amplitudes were $\left|q_{e} E_{B, F}\right| / m_{e} \omega_{p} c=0.1$ in accordance with those observed in the Vlasov simulations. For each calculated electron trajectory the field phases $\varphi_{i}$ were chosen randomly. Figure 11 illustrates the field patterns, and shows an example
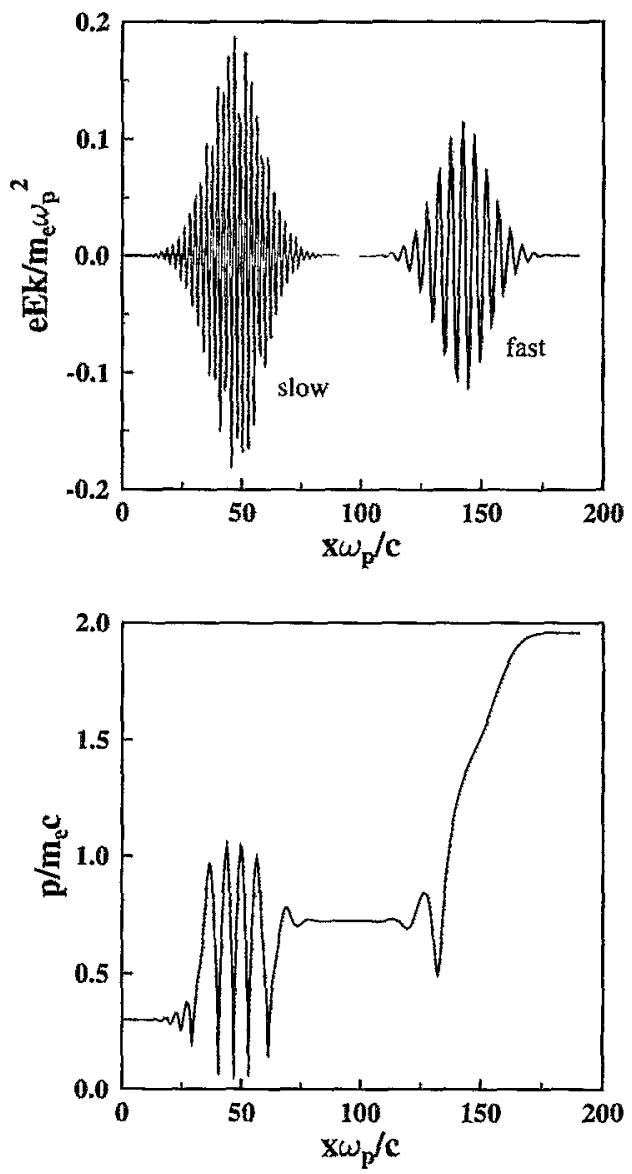

FIG. 11. Momentum of a single test electron travelling through two wave packets with different phase velocities corresponding to SRS-F (fast) and SRS-B (slow) plasma waves (lower frame). The preseribed electrostatic field $\left|q_{e}\right| E(x, t=0) k / m_{e} \omega_{p}^{2}$ is shown in the upper frame. 

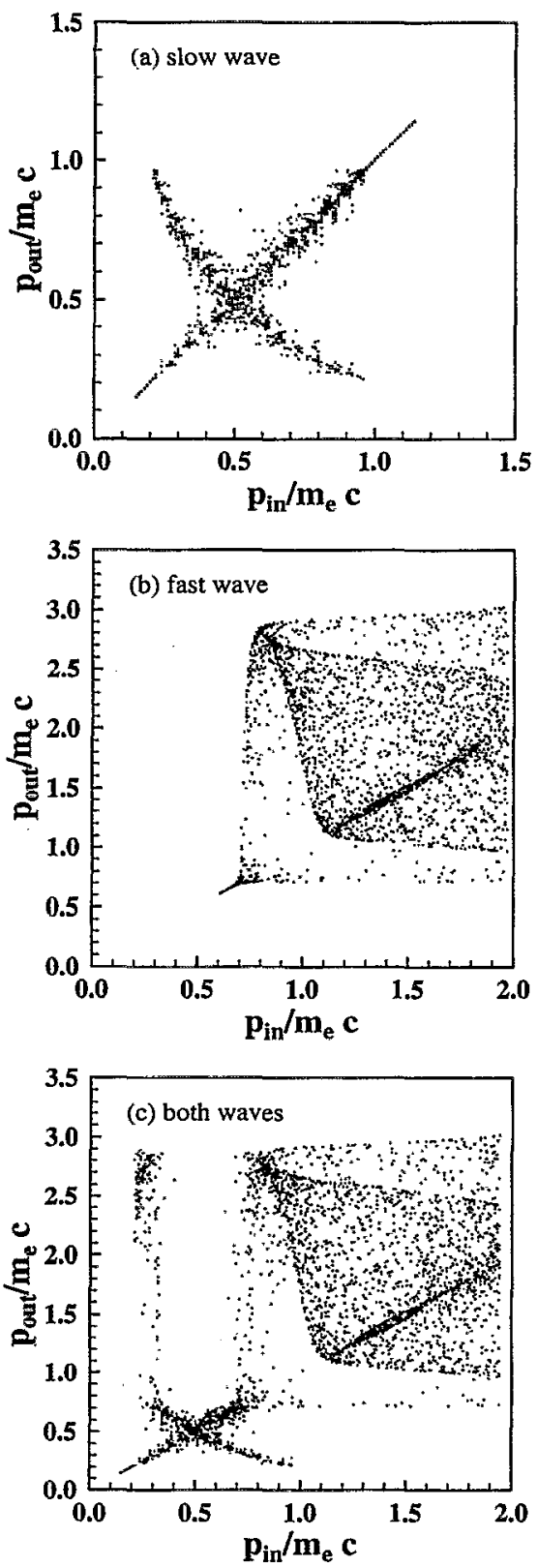

FIG. 12. Final momentum $p_{\text {out }} / m_{e} c$ as a function of incident momentum $p_{\text {in }} / m_{e} c$ for an ensemble of 2000 test electrons with evenly distributed incident momenta. Test electrons propagate (a) through the slow wave packet, (b) through the fast wave packet, and (c) through both spatially separated Gaussian wave packets.

of the, electron trajectory with the initial momentum $p_{\text {in }} / m_{e} c=0.3$. After passing through both wave packets, the final momentum reaches the very high value of $p_{\text {out }} / m_{e} c=1.95$.

To investigate the fast electron generation, we have considered an ensemble of test electrons having an even distribution of initial momenta $p_{\text {in }}$. First, we have calculated the final momenta of the electrons when they only travel through the slow SRS-B like plasma wave. The final momenta $p_{\text {out }}$ form a cross-like structure as is shown in Fig. 12(a). When a similar ensemble of test electrons travels only through the fast SRS-F like plasma wave, the result is quite different as is shown in Fig. 12(b). The difference in the SRS-B and SRS-F

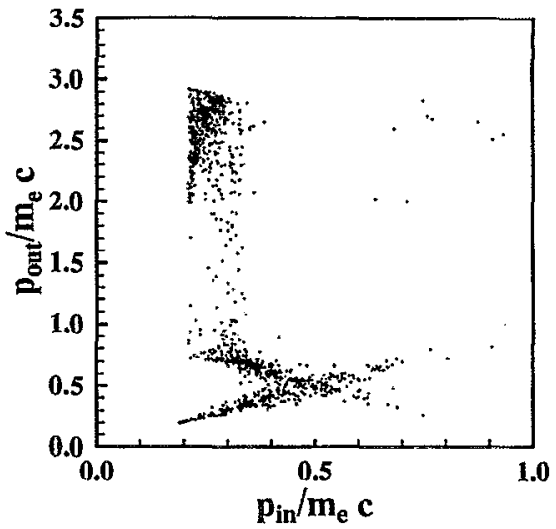

FIG. 13. Final momentum $p_{\text {out }} / m_{e} c$ as a function of incident momentum $p_{\text {in }} / m_{e} c$ for an ensemble of 2000 test electrons with thermally distributed incident momenta. Test electrons propagate through both spatially separated Gaussian wave packets.

patterns arises from the relativistic effects which are dominating in SRS-F. Even in SRS-B, the relativistic effects can be seen as curvature of the cross-like structure of Fig. 12(a).

The combined effect of the two plasma waves on the momenta of the electrons is shown in Fig. 12(c). The island of data points in the upper left-hand corner is due to the two-stage acceleration by the SRS-B and SRS-F plasma waves. It is quite remarkable that the electrons with $p_{\text {in }} / m_{e} c \approx 0.3$ are directly accelerated to $p_{\text {out }} / m_{e} c \simeq 2$ to 3 . SRS-B alone would only produce electrons up to about $p_{\text {out }} / m_{e} c<1$.

In thermal distribution at fusion temperatures, there is only a negligible amount of electrons with momenta $p_{\text {in }} / m_{e} c>0.7$ which are required for an efficient single-step acceleration by SRS-F. Therefore, the synergy between SRS-B and SRS-F leads to an unanticipated amount of very fast electrons. To illustrate this point, we have repeated the previous calculation by choosing the initial momenta of the electrons from the thermal distribution with a temperature of $T_{e}=10 \mathrm{keV}$. In Fig. 13, we see that the scattering pattern caused by SRS-F plasma wave disappears almost completely because of the small amount of hot electrons in the thermal distribution. The island of very fast electrons with the final momenta $p_{\text {out }} / m_{e} c \simeq 2 \ldots 3$ which is generated by the combined action of the two plasma waves becomes now even more striking. This feature can be seen quite clearly in the Vlasov simulation, too. The phase-space plot at $\omega_{p} t=300$ in Fig. 8 shows a free streaming hot population $\left(p / m_{e} c \simeq 2.5\right.$ ) at $x \omega_{p} / c>190$. Most of these electrons have travelled through both plasma wave structures as in the test particle calculation above.

\section{TEST ELECTRONS IN SELF-CONSISTENT FIELDS}

In Sec. IV, we have shown that the Eulerian Vlasov code is able to show strikingly clear pictures of the phase space dynamics, showing impressive agreement with simple orbit theory of steady waves. In an earlier paper, ${ }^{31}$ we have followed the trajectories of individual particles that were trapped and accelerated by the noiseless electrostatic field calculated by the Vlasov code. We have compared these tra- 
jectories with the phase space representation obtained from the Vlasov simulation during the formation of the spiraling vortices in the field of the same plasma wave. The excellent agreement found is an additional indication of the very good performance of the numerical code. These investigations also provide a powerful method for studying the trapping and two-stage acceleration mechanism in the present case because the fields and the trajectories of the particles are free from the statistical fluctuations which are characteristic of the PIC codes.

The trajectories of the "marker" particles are obtained by integrating the equations of characteristics of Eq. (21):

$$
\begin{aligned}
& \frac{d x}{d t}=v_{x}=\frac{p_{x}}{m_{e} \gamma}, \\
& \frac{d p_{x}}{d t}=F(x, t)=q_{e}\left[E_{x}+u_{y}(x, t) B_{z}\right] .
\end{aligned}
$$

The force $F(x, t)$ is directly computed from the results of the Vlasov simulation. The details on how Eqs. (30) and (31) are advanced and the force interpolated have been described in Ref. 31 .

In the Vlasov simulations of Sec. IV, the spectral components of the plasma waves corresponding to SRS-B and SRS-F were well separated (see Fig. 6). Therefore, we were able to separate the electrostatic field components corresponding to SRS-B and SRS-F, respectively (cf. Fig. 7). In the following, the separation of the field components is used in order to analyze the two-stage acceleration of the electrons in the self-consistent field. The analysis makes possible a detailed comparison of the Vlasov simulation results with the test particle calculations of Sec. V.

\section{A. Continuous injection of test electrons}

The first series of "experiments" is performed by continuously injecting test electrons in the plasma near the left boundary at $x=26.5$. Starting at $\omega_{p} t=110,20$ new test electrons are injected in the plasma at each time step, so that at the end of the simulation $\left(\omega_{p} t=340\right)$ the total amount of test electrons is 50000 . The momenta of the test electrons are evenly distributed between $p_{x} / m_{e} c=0.25$ and 0.35 , which is somewhat below the momentum corresponding to the phase velocity of the SRS-B plasma wave $p_{\mathrm{phB}} / m_{e} c=0.50$. The initial momentum was chosen so that it would be possible to see the two-stage acceleration demonstrated in Fig. 12(c). Note that initially the test electrons have a velocity of only about two times the thermal velocity.

We first remove the SRS-F plasma wave from the electrostatic spectrum of the Vlasov simulation described in Sec. IV. This is done by Fourier filtering the electrostatic field at each time step in the wave number space. Thus, the test electrons see only the SRS-B plasma wave whose envelope at $\omega_{p} t=180$ and 300 was shown in Fig. 7. In Fig. 14, the locations of the test electrons in the phase space are shown at times $\omega_{p} t=200,240,280$ and 340 . In the region $20 \leqslant x \omega_{p} / c \leqslant 60$, near the left boundary of the plasma, the test electrons are trapped in the potential of the SRS-B plasma wave (cf. Fig. 7), and form rather clear-cut vortices. Deeper inside the plasma, in the region $x \omega_{p} / c>60$, the field

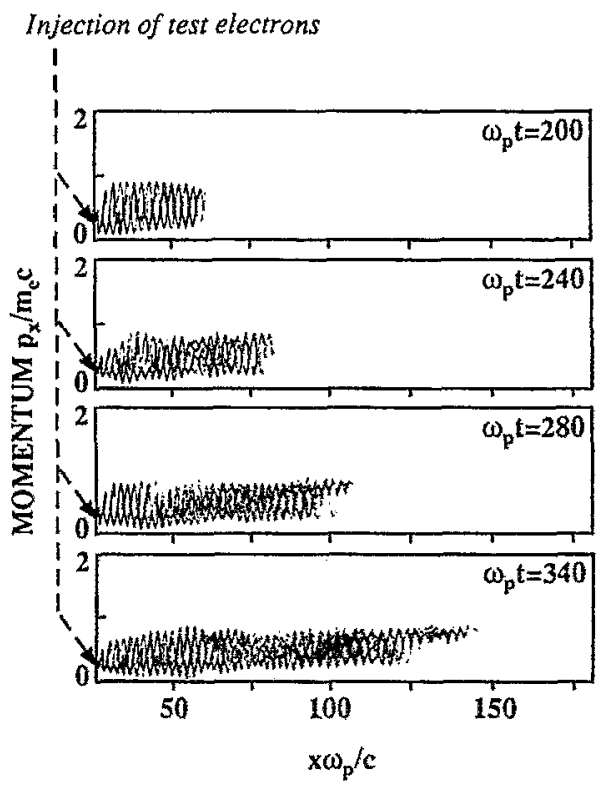

FIG. 14. An ensemble of test electrons entering the plasma at the left boundary is shown at $\omega_{p} t=200,240,280$ and 340 . Twenty test electrons are injected in the plasma per time step during the simulation. The selfconsistent electrostatic field used in the test particle simulation has been obtained from the Vlasov simulation of Sec. IV. The test electrons see only the SRS-B component of the plasma wave because the SRS-F component has been removed from the wave number spectrum by Fourier filtering at each time step.

is decreasing and the electrons are detrapped above the separatrix (see, e.g., Ref. 31). At $\omega_{p} t=280$, this kind of detrapping is just occurring for the test electrons at $x \omega_{p} / c=100$. Later, the behavior of these electrons is ballistic as can be seen in the frame $\omega_{p} t=340$ in the region $x \omega_{p} / c>110$. It is important to note that the final momentum of these electrons does clearly exceed their initial momentum ( $\left.p_{\text {in }} / m_{e} c=0.25-0.35\right)$, and that it is determined by the maximum momentum of the electrons near the upper separatrix.

In Fig. 15, we consider the test electron motion in the fields of SRS-B and SRS-F plasma waves. Recall that the two fields are spatially separated (see Fig. 7). Therefore, the test electrons indeed behave in the same manner at time $\omega_{p} t=200$ in Figs. 14 and 15. Later, however, the fastest electrons are trapped by the SRS-F wave, and further accelerated to high momenta. The interaction of the electrons with the fast SRS-F wave begins at $\omega_{p} t=240$.

It is now interesting to compare the previous results to the situation where the SRS-B plasma wave is absent. In Fig. 16, the SRS-B component has been removed from the electrostatic field by Fourier filtering, and the test electrons see only the SRS-F plasma wave. The initial momentum of the electrons is again $p_{\text {in }} / m_{e} c=0.25-0.35$ as in the previous test particle simulations. Because of the lack of the preacceleration by the SRS-B, no trapping in the potential of the fast SRS-F wave occurs.

\section{B. Momentum change of test electrons}

The second series of "experiments" is performed by following the time evolution of an ensemble of 16000 test 


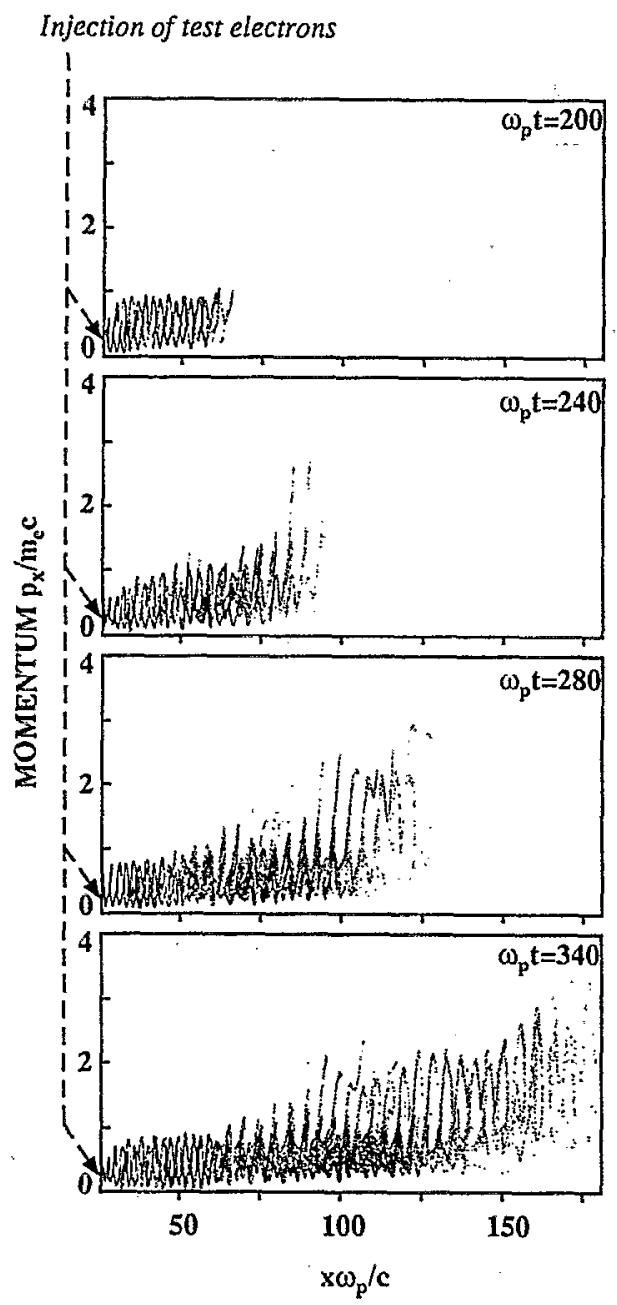

FIG. 15. As in Fig. 14, except that no Fourier filtering of the fields has been performed. Therefore, the test electrons see both the SRS-B and the SRS-F component of the plasma wave.

electrons which are initially evenly distributed in a rectangular box in phase space. The momenta of the electrons are chosen randomly from the interval $0.1 \leqslant p_{x} / m_{e} c \leqslant 1$ and the spatial coordinates from $26 \leqslant x \omega_{p} / c \leqslant 33$, where the spatial length of the box is chosen to be three wavelengths of the SRS-B plasma wave. The time evolution of the test electrons is chosen to start at time $\omega_{p} t=110$ because SRS-B has then already started near the front edge of the plasma. It is of interest to plot again the scattering patterns, where the final momentum of a test electron $p_{\text {out }}$ is plotted versus its initial momentum $p_{\text {in }}$. The final momentum is now measured at the end the simulation, at $\omega_{p} t=340$.

In order to compare the results of the Vlasov simulations to the scattering pattern shown in Fig. 12(a) we have again removed the SRS-F component from the electrostatic field. The scattering pattern obtained when the square of test electrons travels through the remaining SRS-B field is shown in Fig. 17. The cross-like structure in Fig. 17 is in striking agreement with the one found in Fig. 12 even though in the Vlasov simulation the amplitude of the SRS-B field is varying in time when the test electrons are travelling through it.

In Fig. 18, we show the result of a similar experiment

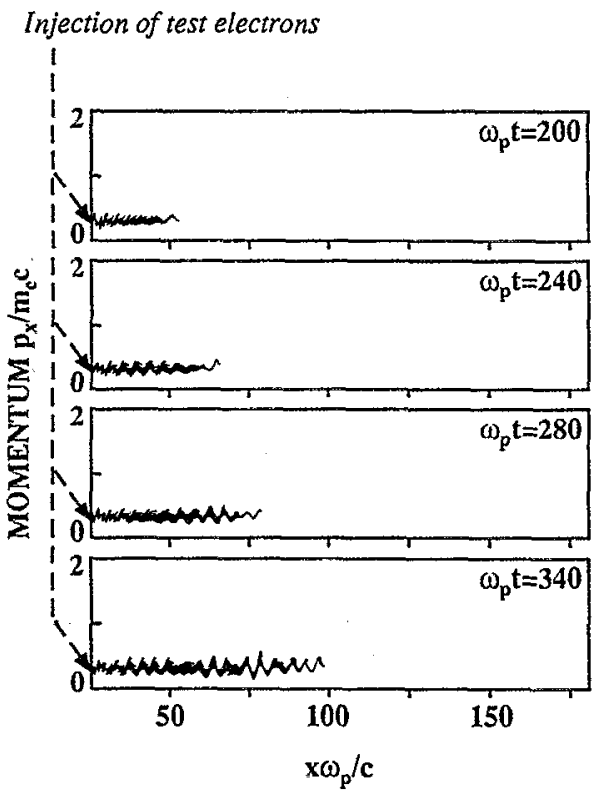

FIG. 16. As in Fig. 14, except that now the SRS-B component of the plasma wave has been removed by Fourier filtering. Therefore, the test electrons see only the SRS-F component of the plasma wave.

where both the SRS-B and the SRS-F plasma waves are present. The main features of Fig. 18 are quite similar to those of Fig. 12(c) but the detailed structures of the scattering pattern have smeared out. There are two reasons for this difference. First, most test electrons are still trapped in the potential well of the SRS-F field at the end of the simulation when the final momentum $p_{\text {out }}$ is measured. These electrons perform oscillatory motion in the high amplitude field, which smears out the scattering pattern. Second, the envelopes of the SRS-B and the SRS-F fields are somewhat overlapping in the Vlasov simulation.

The effect of overlapping of the SRS-B and the SRS-F fields can be studied with the simple test particle model of

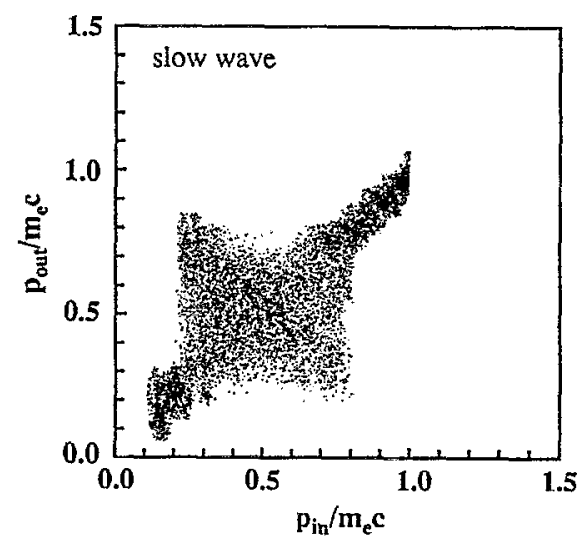

FIG. 17. Final momentum $p_{\text {out }} / m_{e} c$ as a function of incident momentum $p_{\text {in }} / m_{e} c$ for an ensemble of 8000 test electrons with evenly distributed incident momenta. The self-consistent electrostatic field used in the test particle simulation have been obtained from the Vlasov simulation of Sec. IV. The test electrons see only the SRS-B component of the plasma wave because the SRS-F component has been removed from the wave number spectrum by Fourier filtering at each time step. 


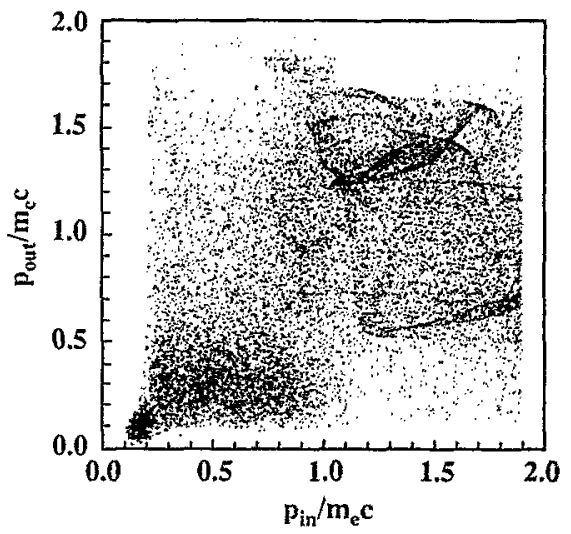

FIG. 18. As in Fig. 17, except that no Fourier filtering of the field has been performed. Therefore, the test electrons see both the SRS-B and the SRS-F component of the plasma wave.

Sec. V by moving the wave packets of Fig. 11 closer to each other. In Fig. 19, the results are shown from a simulation where the spatial separation between the peaks was $25 \omega_{p} / c$. The cross-like momentum pattern at lower velocities is now almost smeared out as in Fig. 18. This is because the electron motion in momentum space becomes diffusionlike when the wave packets are overlapping spatially and the trapping widths are overlapping in the velocity space.

The inherent phase-space resolution of the Eulerian Vlasov code makes it possible to present the detailed structures of phase space shown above. In the more common PIC codes, they would be very coarsely represented by a small number of particles. The essential difficulty in obtaining as accurate phase-space plots with PIC codes as with Eulerian Vlasov codes lies in the number of the test particles in the interesting regions of phase space. One should be able to have a large number of particles in the interesting region without having to provide an enormous number of particles in the uninteresting regions. The present work indicates, however, that the combination of "marker" particles with the noiseless Vlasov solution is a powerful tool for obtaining

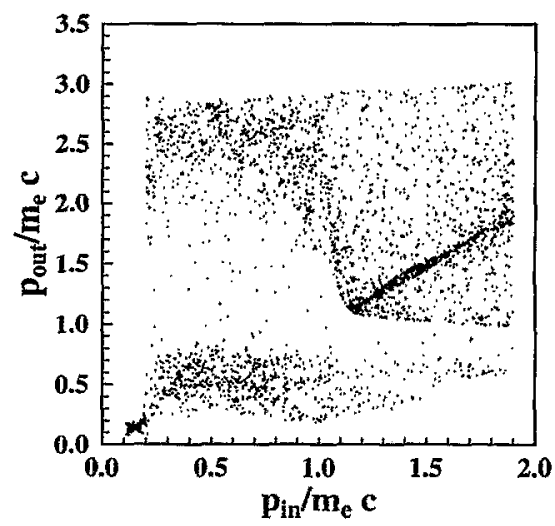

FIG. 19. Final momentum $p_{\text {out }} / m_{e} c$ as a function of incident momentum $p_{\text {in }} / m_{e} c$ for an ensemble of 2000 test electrons with evenly distributed incident momenta. Test electrons propagate through two spatially overlapping prescribed Gaussian wave packets. insight into the trapping and acceleration mechanisms of electrons.

\section{SUMMARY AND DISCUSSION}

The competition between Raman forward and backscattering is an interesting problem because the damping and gain conditions of the two processes can vary considerably. The parameter analysis predicts that there exists a fairly large region in the plasma parameter space where SRS-B and SRS-F may occur simultaneously. In laser-fusion with millimeter size pellets and long pulses, a very long underdense hot plasma surrounds the target. In these conditions, stimulated Raman backscattering is suppressed and forward scattering prevails.

The simultaneous occurrence of SRS-B and SRS-F may lead to a very efficient two-stage acceleration of the plasma electrons. The plasma wave excited by SRS-B has a lower phase velocity so that it interacts with a large number of background electrons preaccelerating them to moderate energies. These electrons can be further accelerated by the fast SRS-F plasma wave, which has its maximum field closer to the rear part of the interaction region.

The relative strength of the forward and backward scattering can be controlled by external beams having frequencies equal to those of the forward or the backward scattered waves. This kind of artificial manipulation of the noise levels could be successful provided that the total gains of the two processes are of the same order of magnitude. The external noise should be broadband and not too intense to avoid the beat-wave like sensitivity for detuning. ${ }^{32}$ This method, which would allow some control over the SRS-B/SRS-F system, would be particularly valuable in current drive of tokamak plasmas by free electron lasers. It would provide a means to control the number and the energy of current-carrying fast electrons.

In our simulations, we have assumed a homogeneous plasma. In the current drive application, the plasma homogeneity is a good approximation because the density does not vary along the magnetic surface. In laser-fusion, very long scale lengths are expected in reactor scale experiments so that the homogeneous plasma model should describe the situation fairly well. In an inhomogeneous plasma, the propagation of the plasma wave may couple the SRS-B and SRS-F systems in a fashion similar to the $2 \omega_{p}$-instability and the half-harmonic generation. ${ }^{18}$ This may lead to enhanced forward scattering or even to an absolute growth of the instability. ${ }^{16,17}$ Weak Landau damping of the SRS-B plasma wave is, however, a prerequisite of the propagation of the plasma wave up along the density gradient.

A weak density gradient is not expected to affect the spatial separation of SRS-B and SRS-F. The main reason for the spatial separation is the wave propagation effect. In SRS-F, all waves propagate in the same direction so that the convective growth requires some distance. In SRS-B, the backscattered wave reaches a high amplitude near the front of the plasma, and therefore the plasma wave is also localized in the same region. A weak density gradient may modify the situation but the fast electron generation is expected to be at least qualitatively similar as in the homogeneous case. 
If the plasma has a strong density gradient, the dynamics of the fast electron generation are expected to change compared to the homogeneous case. The reason for this is that plasma waves with different frequencies will be resonantly driven at each density. Therefore, a broad spectrum of plasma waves will be present at each location, and the clean spatial separation of the almost monochromatic SRS-F and SRS-B plasma waves will not occur. When the electrostatic field contains a large number of modes with different phase velocities, the motion of the electrons in wave potential becomes stochastic. The tail formation in the velocity distribution can in this case be described by the diffusion equation where the diffusion coefficient is obtained from the quasilinear theory. ${ }^{33,34}$ This wave-induced velocity diffusion of electrons and the formation of the fast electron tail have recently been investigated by. Vlasov simulations ${ }^{35}$ where a spectrum of plasma waves was excited by an external electrostatic field. To our knowledge, this phenomena has not been investigated in situations where a broad spectrum of electron plasma waves is excited by SRS in an inhomogeneous plasma.

Our simulations do not take into account the ion motion which starts to modify the wave behavior in a longer time scale. The ion motion makes possible decay or modulational instabilities of the electron plasma waves and stimulated Brillouin scattering of the incident radiation. The SRS-B plasma wave is fairly heavily damped which suppresses the parametric decay of the electron plasma wave especially when the ion acoustic waves are heavily damped, too. ${ }^{36}$ Long-wavelength intense plasma waves generated by SRS-F may become modulationally unstable which may have some effect on the SRS-F part of the fast electron tail. The effect of the ion dynamics on simultaneous SRS-B and SRS-F deserves a more detailed investigation but in such simulations much longer computing times would be required.

Our Vlasov and test particle simulations show that simultaneous Raman backward and forward scattering are able to produce super-hot electrons with $\mathrm{MeV}$ energies. In the front part of the plasma, the SRS-B plasma wave preaccelerates a large number of electrons from the bulk of the distribution. When the electrons propagate deeper inside the plasma, they are further energized by the faster SRS-F plasma wave. An essential feature in this two-stage process is that SRS-B and SRS-F are spatially localized and separated. SRS-B occurs near the front region, and SRS-F occurs much deeper inside the plasma slab. In spatially overlapping wave packets, electron behavior would resemble more a diffusion process than a coherent two-stage acceleration.

In reactor-grade laser fusion experiments, the simultaneous occurrence of SRS-B and SRS-F and the consequent hot electron production make the prevention of SRS particularly important. This kind of two-stage acceleration of electrons could perhaps be applied also to current drive in tokamaks. One could create an analogous situation by two external beams which are spatially non-overlapping and have different frequencies.

\section{ACKNOWLEDGMENTS}

The authors are indebted to the IDRIS centre (Institute for the Development and Resources in Scientific Computation of the CNRS) for computer time allocation on the CrayC98 computer. This research was partly supported by the Finnish Ministry of Trade and Industry.

${ }^{1}$ R. P. Drake, P. E. Young, E. A. Williams, K. Estabrook, W. L. Kruer, B. F. Lasinski, B. Darrow, H. A. Baldis, and T. W. Johnston, Phys. Fluids 31, 1795 (1988).

${ }^{2}$ O. Willi, D. Bassett, A. Giulietti, and S. J. Karttunen, Opt. Commun. 70, 487. (1989).

${ }^{3}$ B. I. Cohen, Comments Plasma Phys. Controlled Fusion 8, 197 (1984).

${ }^{4}$ B. I. Cohen, R. H. Cohen, B. G. Logan, W. McCay Nevins, G. R. Smith, A. V. Kluge, and A. H. Kritz, Nucl. Fusion 28, 1519 (1988).

${ }^{5}$ J. A. Heikkinen, S. J. Karttunen, and R. R. E. Salomaa, Nucl. Fusion 28, 1845 (1988).

${ }^{6}$ M. R. Amin and R. A. Cairns, Nucl. Fusion 30, 327 (1990).

${ }^{7}$ A. Ghizzo, P. Bertrand, M. M. Shoucri, T. W. Johnston, E. Fijalkow, M. R. Feix, and V. V. Demchenko, Nucl. Fusion 32, 45 (1992).

${ }^{8}$ S. J. Karttunen, T. J. H. Pättikangas, R. R. E. Salomaa, and S. K. Sipilä, Nucl. Fusion 31, 1079 (1991).

${ }^{9}$ P. Bertrand, A. Ghizzo, S. J. Karttunen, T. J. H. Pättikangas, R. R. E. Salomaa, and M. Shoucri, Phys. Fluids B 4, 3590 (1992).

${ }^{10}$ S. H. Batha, D. S. Montgomery, K. S. Bradley, R. P. Drake, K. Estabrook, and B. A. Remington, Phys. Rev. Lett. 66, 2324 (1991).

${ }^{11}$ S. H. Batha, K. S. Bradley, R. P. Drake, K. Estabrook, W. L. Kruer, D. S. Montgomery, and B. A. Remington, Phys. Plasmas 1, 1985 (1994).

${ }^{12}$ K. Estabrook and W. L. Kruer, Phys. Fluids 26, 1892 (1983).

${ }^{13}$ S. J. Karttunen and R. R. E. Salomaa, Laser Part. Beams 10, 75 (1992).

${ }^{14}$ P. Bertrand, A. Ghizzo, S. J. Karttunen, T. J. H. Pättikangas, R. R. E. Salomaa, and M. Shoucri, Phys. Rev. E 49, 5656 (1994).

${ }^{15}$ S. J. Karttunen, T. J. H. Pättikangas, and R. R. E. Salomaa, Plasma Phys. Controlled Fusion 36, 657 (1994).

${ }^{16}$ P. Koch and E. A. Williams, Phys. Fluids 27, 2346 (1984).

${ }^{17}$ D. M. Villeneuve and H. A. Baldis, Phys. Fluids 31, 1790 (1988).

${ }^{18}$ S. J. Karttunen, Laser Part. Beams 3, 157 (1985).

${ }^{19}$ D. W. Forslund, J. M. Kindel, and E. L. Lindman, Phys. Fluids 18, 1002 (1975).

${ }^{20}$ A. Sjölund and L. Stenflo, Z. Phys. 204, 211 (1967).

${ }^{21} \mathrm{H}$. Wilhelmsson, J. Plasma Phys. 3; 215 (1969).

${ }^{22}$ V. Fuchs, Phys. Fluids 19, 1554 (1976).

${ }^{23}$ N. M. Kroll, J. Appl. Phys. 36, 34 (1965).

${ }^{24}$ Note that the corresponding expression given on p. 88 of Ref. 13 is erroneous.

${ }^{25}$ D. W. Forslund, J. M. Kindel, and E. L. Lindman, Phys. Fluids 18, 1017 (1975).

${ }^{26} \mathrm{G}$. Bonnaud and C. Reisse, Nucl, Fusion 26, 633 (1986).

${ }^{27}$ G. Bonnaud, Plasma Phys. Controlled Fusion 29, 573 (1987).

${ }^{28}$ A. Ghizzo, P. Bertrand, M. M. Shoucri, T. W. Johnston, E. Fijalkow, and M. R. Feix, J. Comput. Phys. 90, 431 (1990).

${ }^{29}$ P. Bertrand, A. Ghizzo, T. W. Johnston, M. Shoucri, E. Fijalkow, and M. R. Feix, Phys. Fluids B 2, 1028 (1990).

${ }^{30}$ V. Fuchs, V. Krapchev, A. Ram, and A. Bers, Physica D 14, 141 (1985).

${ }^{31}$ A. Ghizzo, M. Shoucri, P. Bertrand, T. Johnston, and J. Lebas, J. Comput. Phys. 108, 373 (1993).

${ }^{32}$ S. J. Karttunen and R. R. E. Salomaa, Phys. Scr. 39, 741 (1989).

${ }^{33}$ A. A. Vedenov, E. P. Velikhov, and R. Z. Sagdeev, Nucl. Fusion 1, 82 (1961).

${ }^{34}$ W. E. Drummond and D. Pines; Nucl. Fusion Suppl. 3, 1049 (1962).

${ }^{35}$ J. T. Berndtson, J. A. Heikkinen, S. J. Karttunen, T. J. H. Pättikangas, and R. R. E. Salomaa, Plasma Phys. Controlled Fusion 36, 57 (1994).

${ }^{36}$ J. A. Heikkinen and S. J. Karttunen, Phys. Fluids 29, 1291 (1986). 